(C) (C) 2017 IEEE. Personal use of this material is permitted. Permission from IEEE must be obtained for all other uses, in any current or future media, including reprinting/republishing this material for advertising or promotional purposes, creating new collective works, for resale or redistribution to servers or lists, or reuse of any copyrighted component of this work in other works

This is the authors' post-print version of the journal paper published in IEEE JOURNAL OF SELECTED TOPICS IN APPLIED EARTH OBSERVATIONS AND REMOTE SENSING. Citations to this paper should be as follows: A. Di Simone, H. Park, D. Riccio, and A. Camps, "Sea Target Detection Using Spaceborne GNSS-R Delay-Doppler Maps: Theory and Experimental Proof of Concept Using TDS-1 Data," IEEE J. Sel. Top. Appl. Earth Obs. Remote Sens., vol. 10, no. 9, 2017. doi: 10.1109/JSTARS.2017.2705350 


\title{
Sea Target Detection Using Spaceborne GNSS-R Delay-Doppler Maps: Theory and Experimental Proof of Concept Using TDS-1 Data
}

\author{
Alessio Di Simone, Hyuk Park, Senior Member, IEEE, Daniele Riccio, Fellow, IEEE, \\ and Adriano Camps, Fellow, IEEE
}

\begin{abstract}
This study addresses a novel application of global navigation satellite system-reflectometry (GNSS-R) delay-Doppler maps (DDMs), namely sea target detection. In contrast with other competing remote sensing technologies, such as synthetic aperture radar and optical systems, typically exploited in the field of sea target detection, GNSS-R systems could be employed as satellite constellations, so as to fulfill the temporal requirements for near real-time ships and sea ice sheets monitoring. In this study, the revisit time offered by GNSS-R systems is quantitatively evaluated by means of a simulation analysis, in which three different realistic GNSS-R missions are simulated and analyzed. Then, a sea target detection algorithm from spaceborne GNSS-R DDMs is described and assessed. The algorithm is based on a sea clutter compensation step and uses an adaptive threshold to take into account spatial variations in the sea background and/or noise statistics. Finally, the sea target detector algorithm is tested and validated for the first time ever using experimental GNSS-R data from the U.K. TechDemoSat-1 dataset. Performance is assessed by providing the receiver operating characteristic curves, and some preliminary experimental results are presented.
\end{abstract}

Index Terms-Constant false alarm rate (CFAR), global navigation satellite system-reflectometry (GNSS-R), maritime surveillance, sea state, sea target detection.

\section{INTRODUCTION}

$\mathbf{S}$ EA target monitoring is of key importance in the field of global monitoring of environment, maritime security, and surveillance. Accurate monitoring, continuous mapping, and large-scale analysis of sea surface, sea-ice sheets, and ship traffic are essential for activities as diverse as engineering, safety, travel, agriculture, recreation, and commerce. Real-time maritime surveillance and ship monitoring are relevant in a wide range of applications, such as clandestine activities control,

Manuscript received November 13, 2016; revised March 14, 2017 and May 4, 2017; accepted May 5, 2017. (Corresponding author: Alessio Di Simone.)

A. Di Simone is with the Dipartimento di Ingegneria Elettrica e delle Tecnologie dell'Informazione, Università degli Studi di Napoli Federico II, 80125 Napoli, Italy (e-mail: alessio.disimone@unina.it).

H. Park and A. Camps are with the Department of Signal Theory and Communications, Universitat Politècnica de Catalunya, 08034 Barcelona, Spain (e-mail: park.hyuk@tsc.upc.edu; camps@tsc.upc.edu).

D. Riccio is with the Dipartimento di Ingegneria Elettrica e delle Tecnologie dell'Informazione, Università degli Studi di Napoli Federico II, 80125 Napoli, Italy (e-mail: daniele.riccio@unina.it).

Color versions of one or more of the figures in this paper are available online at http://ieeexplore.ieee.org.

Digital Object Identifier 10.1109/JSTARS.2017.2705350 naval warfare, traffic surveillance, oil discharge, and sea pollution monitoring [1], [2]. In particular, in recent years, because of the decrease in fishery resources in the world, ship detection has become much more important for effective and efficient illegal fishing activities monitoring.

On the other hand, ice sheet mapping is fundamental to analyze climate dynamics and evaluate human-induced climate changes. Currently, most of this information is gathered with very high accuracy by means of ground-based stations and networks or with coarse resolution microwave radiometers. Despite the high accuracy provided and the well-assessed technology, in-situ measurements only provide local information. The spatial coverage provided by ground-based networks is generally limited to low elevation regions of the Northern Hemisphere mid-latitudes and to snow course in mountainous regions [3]. In order to address accurate ice sheet mapping on a global scale, space-borne measurements from satellite constellations come into play. Detailed coarse-resolution daily maps of polar ice cover derived from remote sensors are now publicly available on the website of the U.S. National Snow and Ice Center [4].

Concerning international trade, more than $80 \%$ of the global trade and almost $90 \%$ of freight trade external to the European Union are seaborne [5], [6]. The civil seaborne traffic transports more than 400 million passengers in European ports each year [5]. The large density of worldwide maritime traffic in 2015 is shown in Fig. 1. The automatic identification system (AIS) is commonly used for coastal-traffic monitoring, and it provides deep information about the ship traffic, such as position, velocity, and route, and a unique reference, such as the International Maritime Organization number. However, coastal AISs are limited in their coverage, thus covering up to $40 \mathrm{~km}$ off the coast [1]. To overcome this limitation, very recently, the AIS technology has been mounted on-board spaceborne platforms, such as the SatAIS launched in 2011 by the German Aerospace Centre [1] or the planned U.K. NovaSAR-S [7] to be launched in the next years. However, the main drawback of the AIS protocol in the field of maritime security and surveillance is the need for collaborating ships and ships equipped with correctly operating AIS facilities on board. Remote sensing imagery gives the possibility to overcome these limits, thus allowing for the detection and tracking of noncooperative ships and small ships without an AIS system on board [8]. 


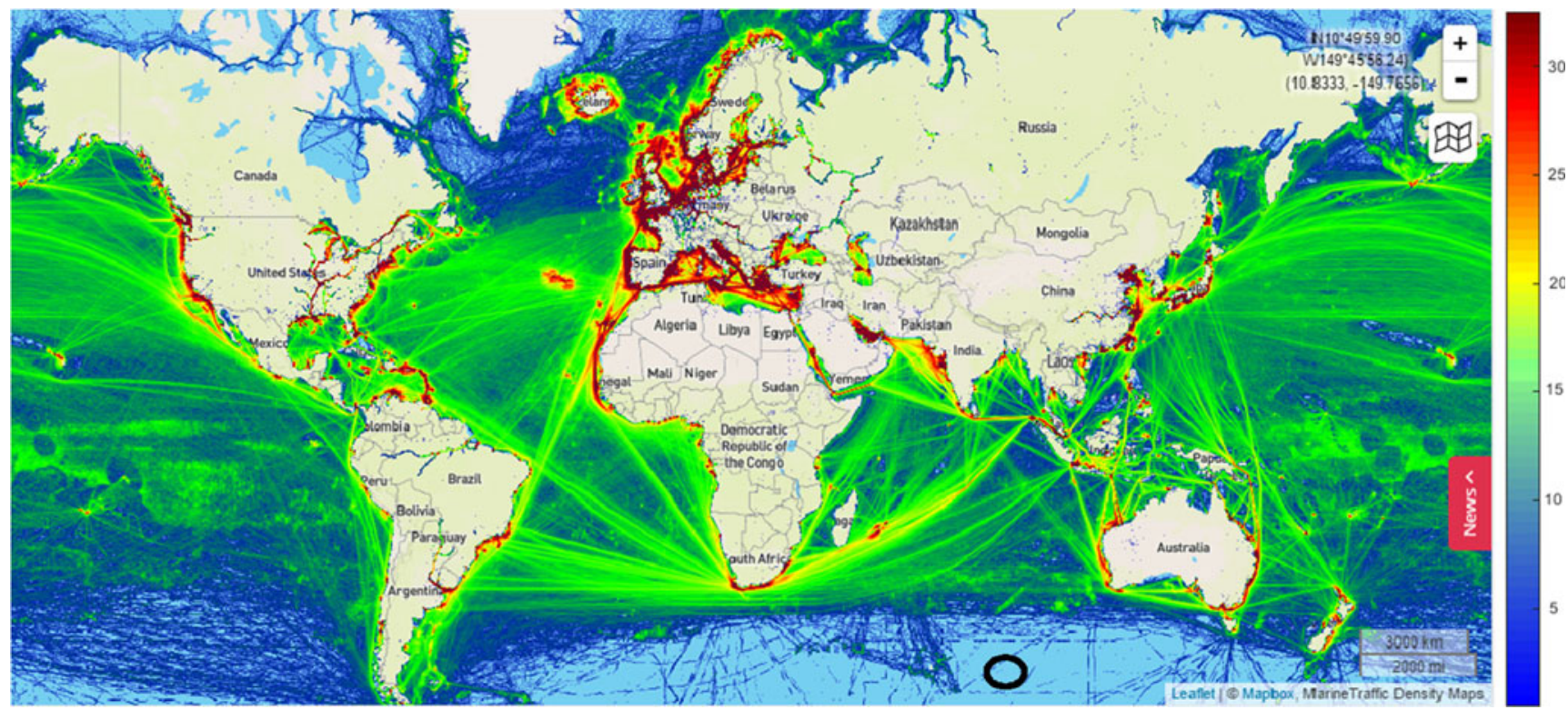

Fig. 1. 2015 worldwide maritime traffic density map. The density is evaluated as the number of ships per $1^{\circ} \times 1^{\circ}$ grid cell per day (from www.marinetraffic.com). The black circle indicates the region selected in the third (target-free) scenario. Cyan background indicates regions with no sea traffic.

During the last three decades, remote sensing has acquired an increasing appeal in the international scientific community owing to its wide potentialities. An increasing number of applications have been conceived and developed after the launch of new high-resolution sensors. Among them, optical and synthetic aperture radar (SAR) systems have been intensively exploited in the field of ship detection. An extensive literature on sea target detection and classification from optical and SAR data exists [1], [2], [9], [10]; a comprehensive state-of-the-art review report about ship detection from SAR data can be found in [11]. Although comprehensive information can be acquired about the target state by means of AIS technology, the International Maritime Organization's International Convention for the Safety of Life at Sea only requires AIS to be fitted aboard international voyaging ships with gross tonnage of 300 tons or more, and all passenger ships regardless of size [8]. Therefore, detection of small ships and ships in open sea is currently a compelling application of remote sensing systems.

Owing to their all-day and all-weather imaging capabilities, SAR systems represent the most exploited remote sensing technology for ship detection, arousing even more interest after the launch of the new-generation high-resolution sensors, such as TerraSAR-X, RADARSAT-2, and the Sentinel-1 and COSMOSkyMed constellations. Very accurate detection rate and positioning are currently addressed by most of the existing stateof-the-art ship detection techniques. However, the time resolution, i.e., revisit time, of SAR and optical sensors represents the main limitation for ship/ice detection applications. With a limited number of satellites, the revisit cycle is quite long and cannot meet the requirements for real-time ship and ice sheets monitoring [10], [12]. A time resolution on the order of hours is required for ship detection [12]. The World Meteorological Organization indicates a revisit time on the order of hours for sea-ice cover concerning the Global Numerical Weather Prediction, climate monitoring, and ocean applications [13].

A revisit time on the order of days is provided by current optical and SAR sensors but the COSMO-SkyMed constellation that ensures a revisit time up to 12 hours. However, the end of life of COSMO-SkyMed is expected to be at the end of 2017 [14].

Global navigation satellite system-reflectometry (GNSS-R) represents a relatively new remote sensing technology, first discussed in the late 1980s for scatterometry purposes [15]. It is based on the acquisition and processing of GNSS signals of opportunity scattered from the Earth's surface. So far, one of the main applications of GNSS-R is sea state estimation and monitoring, and several approaches and techniques to estimate the local wind speed from GNSS-R observables have been developed in the last years [16]-[19]. Other very recent applications of the GNSS-R technology concern the surface scattering coefficient retrieval [20], [21], ocean topography [22], oil slick detection [23], [24], and tsunami detection [25], [26]. Due to the absence of a transmitter module, GNSS-R payloads can be mounted on nano- or small-satellites - as the recently launched ${ }^{3}$ Cat-2 satellite by UPC [27] — with the potential to be deployed in wide constellations. Consequently, as showed further in this work, spaceborne GNSS-R systems give the chance to dramatically reduce the revisit time with respect to other remote sensing technologies, such as SAR and optical satellites, thus fulfilling the time resolution requirements for real-time maritime traffic surveillance and ship detection. A partial list of advantages and drawbacks of the aforementioned remote sensing technologies for sea target detection purposes is presented in Table I.

GNSS signals reflected from ice were correctly identified and measured using the UK DMC experiment on February 4, 2005 over the Kuskowkwim Bay Alaska [28], [29]. Earth-reflected global positioning system (GPS) L-band signals 
TABLE I

ADVANTAGES AND DRAWBACKS OF SAR, OptICAL, AND GNSS-R SYSTEMS FOR SEA TARGET DETECTION

\begin{tabular}{|c|c|c|}
\hline & Pros & Cons \\
\hline SAR & $\begin{array}{l}\text { - Independence on weather and light conditions. } \\
\text { - Potential exploitation of multipolarization, multifrequency data. } \\
\text { - Very high spatial resolution (up to } 1 \mathrm{~m} \text { ). }\end{array}$ & $\begin{array}{l}\text { - Active systems (huge cost and size). } \\
\text { - Sensitivity to speckle and sea state, with increasing frequency [53]. } \\
\text { - Difficult visual interpretation. } \\
\text { - Difficult detection of non-metallic (e.g., wooden) targets. } \\
\text { - High revisit time*. } \\
\text { - Large number of false alarms due to the high non-homogeneity of oceanic SAR } \\
\text { imagery [12]. }\end{array}$ \\
\hline Optical & $\begin{array}{l}\text { - Very high spatial resolution (up to } 0.5 \mathrm{~m} \text { ). } \\
\text { - Relatively cheap. } \\
\text { - Suited to hyperspectral imaging. } \\
\text { - Easy to interpret (no expert user needed). }\end{array}$ & $\begin{array}{l}\text { - Sensitivity to sea clutter. } \\
\text { - Unavailable during night and cloudy days. } \\
\text { - High revisit time*. } \\
\text { - The large amount of data prevent the use in real time. }\end{array}$ \\
\hline GNSS-R & $\begin{array}{l}\text { - Worldwide coverage on nearly real time. } \\
\text { - Compact, low-power, light-weight and cheap. } \\
\text { - All-weather, all-time capabilities. } \\
\text { - Ability of counter the attack of antiradiation missiles due to the bistatic } \\
\text { configuration. } \\
\text { - Exploitation of pre-existing transmitters. } \\
\text { - Very low revisit time. }\end{array}$ & $\begin{array}{l}\text { - Low spatial resolution (on the order of km). } \\
\text { •Not yet extensively studied and assessed. }\end{array}$ \\
\hline
\end{tabular}

* TerraSAR-X: 11 days [54]. COSMO-SkyMed: up to $12 \mathrm{~h}$ (4.5h on average); End of Life expected at the end of 2017 [14]. Sentinel-1: 1-3 days [55]. SPOT: 1-3 days (in cloud-free condition) [56]. LANDSAT: 16 days [57]. Sentinel-2: 5 days [58]. Required update time for ship detection on the order of hours [12].

exploited by GNSS-R systems deeply penetrate in ice, allowing for analysis of snowpack internal structures and characteristics, such as thickness and accumulation rates [30]. In contrast with the typical $\mathrm{Ku}$ - and $\mathrm{C}$-bands used in ice sheets analysis from SAR data, the low-frequency GNSS signals can penetrate up to $100 \mathrm{~m}$ in the ice, allowing for ice investigation on the millennium scale [30].

Thanks to the short revisit time and high global coverage of GNSS-R constellations, the sea target detection problem is expected to benefit from GNSS-R imagery. However, these very recent topics have not been properly and deeply investigated yet, and very few works focusing especially on the assessment and feasibility of ship detection from GNSS-R observables can be found in the related literature [31]-[35]. In [31], the possibility to detect ships from GNSS reflected signals is analyzed by means of an experimental airborne mission. In [32], the intriguing chance to detect sea targets from a delay-Doppler map (DDM) acquired in a backscattering configuration is sketched. However, some limiting hypotheses are required due to the particular geometric configuration between the target, the transmitter, and the receiver. In [33], the feasibility of sea target detection from spaceborne GNSS-R DDMs is demonstrated for different target sizes and sea state conditions. A spatial filter based on steerable antenna beams is proposed to solve for the mapping ambiguity. The work in [34] introduces a new constant false alarm rate (CFAR) method for ship object detection from GPS$\mathrm{R}$ correlation power spectra. However, few details are provided about the ship detection algorithm and the algorithm validation.

Several ideas and hints, such as backscattering configuration, sea clutter compensation, and CFAR approaches, have been proposed so far in the literature to perform sea target detection from GNSS-R observables; however, an organic and systematic proposal and validation of a sea target detection algorithm from DDMs represent the current main gap of the state of the art.
In this work, the revisit time achievable with spaceborne GNSS-R systems is analyzed and presented by means of software simulations. The revisit time is evaluated as a function of the number of satellites and channels and guideline graphs are provided and discussed. Furthermore, a sea target detection algorithm from spaceborne GNSS-R observables is presented, described, and validated with actual U.K. TechDemoSat-1 (TDS-1) data. The performance of the technique is assessed through the receiver operating characteristics (ROC) curves.

To summarize, the main contributions of this work are the following:

1) numerical analysis of the revisit time provided by GNSS$\mathrm{R}$ constellations by means of realistic simulated missions (Section II);

2) derivation and implementation of a sea target detection algorithm from spaceborne GNSS-R DDMs (Section III);

3) validation of the algorithm using actual GNSS-R data (Section IV).

Conclusions and future recommendations are in Section V.

\section{REvisit TIME}

The major limiting factor for the practical application of both SAR and optical imagery in the field of maritime surveillance comes from the relatively high revisit time, as shown in Table I.

In contrast to other remote sensing approaches and systems, GNSS-R offers a significant flexibility in terms of costs, weight, and performance, as well as a much faster mission design phase. Revisit time requirements for nearly real-time maritime surveillance can be much easily fulfilled with GNSS-R small satellites and several constellations can be put into orbit at contained costs. This allows GNSS-R offering a revisit time sufficiently low for near real-time ship and ice monitoring purposes. 
TABLE II

ORBITAL PARAMETERS FOR GPS, GALILEO, GLONASS, AND BEIDOU-2 GNSSS

\begin{tabular}{lllll}
\hline \hline & GPS & Galileo & GLONASS & BeiDou-2 \\
\hline Number of orbital planes & 6 & 3 & 3 & 3 \\
Number of Satellites & 24 & 27 & 24 & $35(5 \mathrm{GEO})$ \\
Satellite Altitude [km] & 20180 & 23222 & 19100 & 21150 \\
Orbit inclination [degree] & $55^{\circ}$ & $56^{\circ}$ & $64.8^{\circ}$ & $55.5^{\circ}$ \\
\hline \hline
\end{tabular}

The ongoing cyclone GNSS (CYGNSS) mission by NASA devoted to hurricane forecasting consists of eight small satellites and will provide frequent and accurate measurements of ocean surface winds with a revisit time of $2.8 \mathrm{~h}$ (median) and $7.2 \mathrm{~h}$ (mean) over the full $\pm 35^{\circ}$ using only four parallel measurements [36].

Many variables influence the revisit time of a GNSS-R system. Some of them, such as the glistening zone size and the specular reflection point position, cannot be determined deterministically without a complete knowledge of the actual sea state and of the transmitter/receiver geometry. This will invoke for a statistical modeling of the revisit time, since some influencing parameters need to be statistically described. Alternatively, time resolution of such systems can be analyzed by means of mission simulation studies. Under appropriate hypotheses primarily regarding the duration of the time period simulated, some statistical descriptors can be inferred from the numeric simulation as well.

In this paper, the second approach is followed, leaving the statistical approach to future research activities. The revisit time provided by GNSS-R systems is evaluated by means of the commercial software AGI Systems Tool Kit.

In this simulation study, the dependence of the revisit time of GNSS-R constellations on the number of satellites and the parallel channels is considered. In particular, three different scenarios have been analyzed. In each scenario, (up to) four eight-satellite constellations of GNSS-R instruments on a $98^{\circ}$-inclined equatorial circular orbit at $500-\mathrm{km}$ attitude have been considered; each GNSS-R satellite is equipped with (up to) 16 parallel tracking channels acquiring signals from the GPS only (Scenario 1), GPS and Galileo (Scenario 2), GPS, Galileo, GLONASS, and BeiDou-2 (Scenario 3). Such constellations provide a global coverage to allow for sea target detection at high latitudes as well and can be implemented based on small satellite platforms, such as ${ }^{3}$ Cat-2 [27], [37]. Table II lists the main orbital parameters for the four considered GNSS constellations, while Table III lists the main parameters of the three considered scenarios.

The revisit time has been evaluated by simulating four-day missions with a time step of $120 \mathrm{~s}$, successively interpolated to $60 \mathrm{~s}$ in MATLAB. Earth's surface has been divided in a regular grid in latitude-longitude with a $1^{\circ}$ spacing in both latitude and longitude, corresponding to a $120 \times 120 \mathrm{~km}^{2}$ cell at the Equator and a $120 \times 40 \mathrm{~km}^{2}$ cell at $70{ }^{\circ} \mathrm{N}$. The specular point position is evaluated from the transmitter and receiver positions by means of the Newton-Raphson method, where the WGS-84 is used to model Earth's surface [27], [37]. The revisit time
TABLE III

ORBITAL PARAMETERS OF THE CONSIDERED SCENARIOS

\begin{tabular}{llll}
\hline \hline & Scenario 1 & Scenario 2 & Scenario 3 \\
\hline Altitude [km] & 500 & 500 & 500 \\
Inclination [degree] & $98^{\circ}$ & $98^{\circ}$ & $98^{\circ}$ \\
Orbit type & Circular & Circular & Circular \\
Number of satellites & 32 & 32 & 32 \\
Number of parallel & 16 & 16 & 16 \\
channels & & GPS, Galileo & GPS, Galileo, GLONASS, \\
GNSS systems tracked & GPS & & BeiDou-2 \\
& & &
\end{tabular}

achieved in the three considered scenarios is shown in Figs. 24 , respectively, where (a) the average, (b) the median, and (c) the standard deviation of the revisit time computed in the covered areas are shown as a function of the following variables: the number of tracking channels (i.e., different transmitting space vehicles: 1 to 16) mounted on board GNSS-R instruments; the number of GNSS-R satellites $(1,8,16,24,32)$ considered. Using few GNSS-R satellites leads to time resolution not far from that provided by the most recent SAR and optical missions (see Table I), especially in the case of only few parallel tracking channels (see Fig. 2). In order to lower the revisit time, two solutions are achievable, namely increasing the constellation size of GNSS-R instruments, and/or increasing the number of receiving (tracking) channels per receiver. A minimum mean revisit time of $5 \mathrm{~h}$ and $37 \mathrm{~min}$ is achieved in scenario 1 when using 32 GNSS-R satellites equipped with 16 receiving channels. In both solutions, a reduction of the revisit time is allowed by a higher number of glistening zones simultaneously tracked. The fundamental difference between the two solutions lies in the revisit time improvement allowed. Indeed, regarding the first solution, any desired time resolution can be achieved, at least in principle, by considering a sufficiently large GNSS-R constellation size. However, the improvement of the revisit time allowed by additional GNSS-R satellites diminishes as the constellation size increases, as shown in Fig. 2, in which a relative improvement of about $42 \%$ and $23 \%$ is experienced in the average revisit time when passing from 8 to 16 GNSS-R satellites and from 24 to 32 GNSS-R satellites, respectively. On the other side, the limited number of GNSS stations accessible by the GNSS-R satellite at the same time leads to a limited improvement of the revisit time by increasing the number of parallel measurements. In other words, a further increasing of a sufficiently high number of parallel tracking channels would not imply the acquisition of new Earth-reflected signals. As a result, the revisit time exhibits a plateau as a function of the number of parallel measurements for any fixed GNSS-R constellation size and scenario. For instance, in scenario 1, in which only the GPS stations can be tracked, the revisit time does not exhibit any further significant improvement when using more than six receiving channels (see Fig. 2). If further reductions of the revisit time are required/desirable, the capability to track more GNSS transmitters instead of the introduction of further parallel channels should be taken into account in the design phase of the GNSS-R. Indeed, the higher the efficiency in the exploitation of the parallel channels. In scenario 

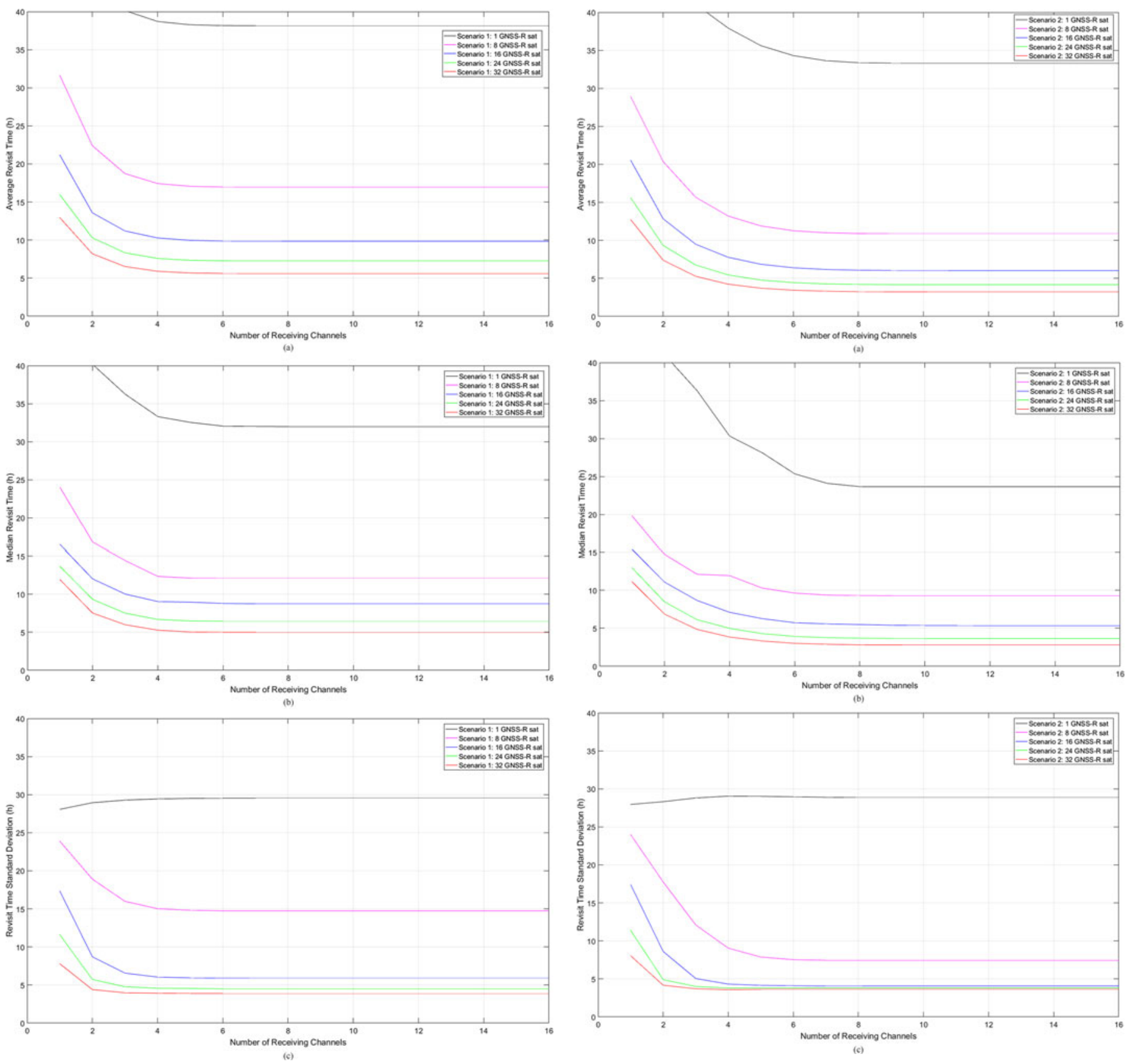

Fig. 2. (a) Mean, (b) median, and (c) standard deviation of the revisit time in hours versus number of receiving channels considering one (black line), eight (magenta line), 16 (blue line), 24 (green line), and 32 (red line) GNSS-R satellites. Only GPS stations can be tracked.

2, the possibility to track also Galileo satellites leads to a minimum mean revisit time of $3 \mathrm{~h}$ and $13 \mathrm{~min}$ with an improvement of $42 \%$ with respect to scenario 1; up to eight GNSS transmitters can be accessed on average at the same time. The higher number of GNSS transmitters accessible simultaneously allows for a more homogeneity of the revisit time as well, as shown in Fig. 3(c). Even lower revisit time can be achieved on average by tracking GPS, Galileo, GLONASS, and BeiDou-2 as in scenario 3 , in which a minimum mean revisit time of $2 \mathrm{~h}$ and 14 min is achieved with an improvement of about $60 \%$ with

Fig. 3. (a) Mean, (b) median, and (c) standard deviation of the revisit time in hours versus number of receiving channels considering one (black line), eight (magenta line), 16 (blue line), 24 (green line), and 32 (red line) GNSS-R satellites. GPS and Galileo stations can be tracked.

respect scenario 1 . In this scenario, no further lowering of the revisit time is experienced when using more than 12 receiving channels.

Fig. 5 shows the average revisit time in scenarios 1 (solid lines) and 3 (dash-dotted lines) as a function of the receiving channels for one (black), 16 (blue), and 32 (red) GNSS-R satellites. As shown in the graph, the benefits of tracking multiple GNSSs are appreciable in the case of a sufficiently high number of tracking channels (compare the solid lines relevant to scenario 1 with the corresponding dash-dotted lines of the 

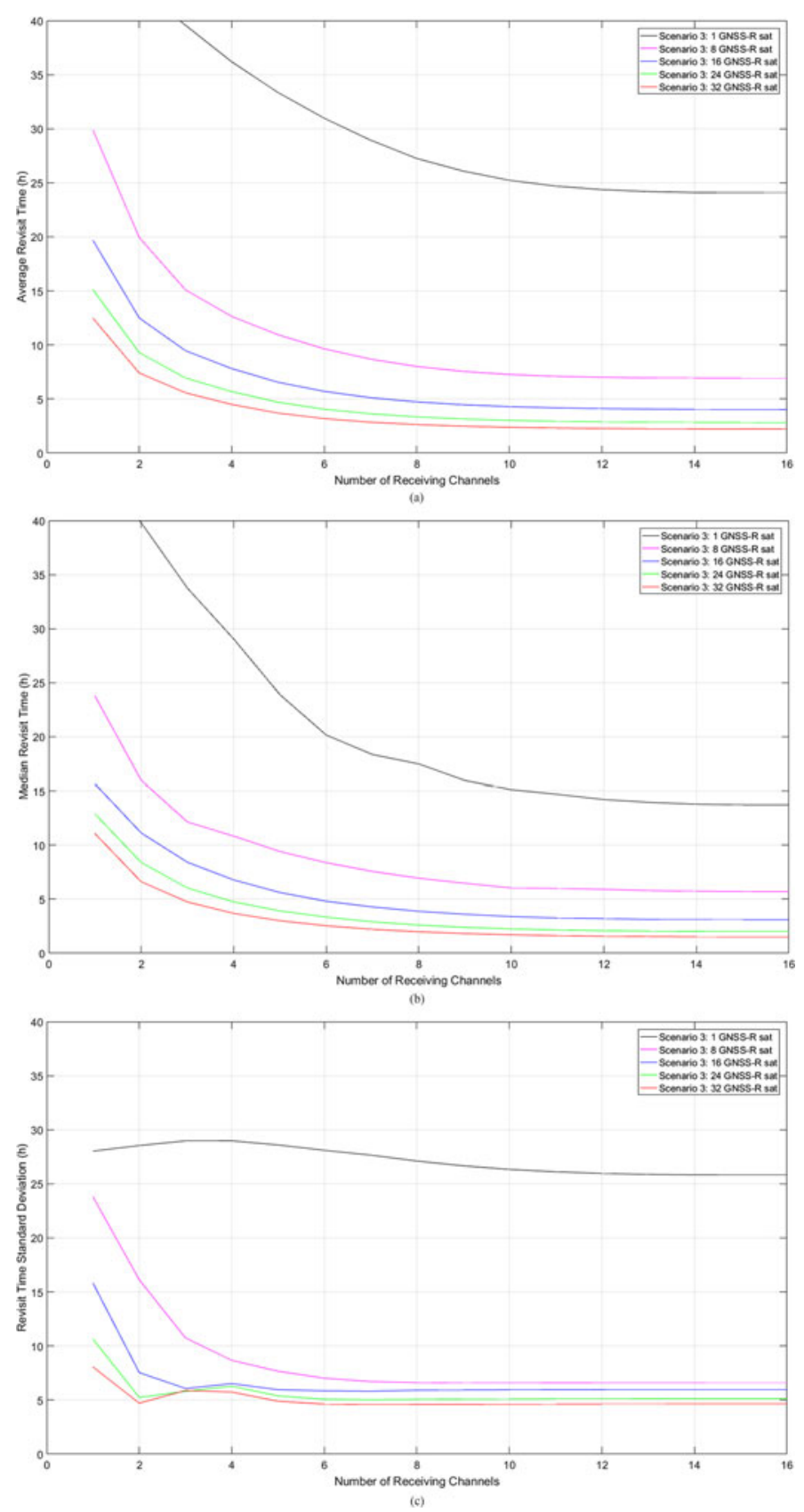

Fig. 4. (a) Mean, (b) median, and (c) standard deviation of the revisit time in hours versus number of receiving channels considering one (black line), eight (magenta line), 16 (blue line), 24 (green line), and 32 (red line) GNSS-R satellites. GPS, Galileo, GLONASS, and BeiDou-2 stations can be tracked.

same color relevant to scenario 3). Indeed, for any fixed number of receiving channels, the possibility to track more GNSS constellations allows for a greater efficiency (defined as the time percentage in which the channel is receiving GNSS signals) of the receiving channels usage. However, in the presence of few receiving channels, even a single GNSS (GPS for instance) constellation ensures a very high efficiency and, consequently, the tracking of additional GNSS constellations (as in Scenario 3) does not lead to a significant improvement of the revisit time. To acquire the increased number of GNSS signals available and,

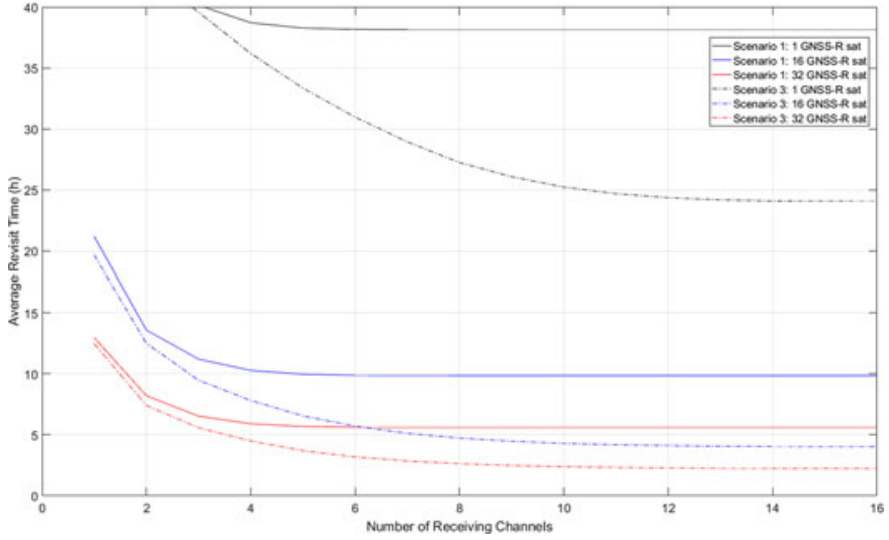

Fig. 5. Mean revisit time as a function of the number of receiving channels in scenario 1 (solid lines-only GPS signals are acquirable) and scenario 3 (dashdotted lines-GPS, Galileo, GLONASS, and BeiDou-2 signals are acquirable) using one (black lines), 16 (blue lines), and 32 (red lines) GNSS-R satellites.

TABLE IV

ReVisit Time StATISTICS For 32 GNSS-R SATELLites Mounting 16 RECEIVING CHANNELS

\begin{tabular}{|c|c|c|c|}
\hline & Scenario 1 & Scenario 2 & Scenario 3 \\
\hline Mean & $5 \mathrm{~h} 37 \mathrm{~m}$ & $3 \mathrm{~h} 13 \mathrm{~m}$ & $2 \mathrm{~h} 14 \mathrm{~m}$ \\
\hline Median & $4 \mathrm{~h} 59 \mathrm{~m}$ & $2 \mathrm{~h} 48 \mathrm{~m}$ & $1 \mathrm{~h} 31 \mathrm{~m}$ \\
\hline Standard deviation & $3 \mathrm{~h} 53 \mathrm{~m}$ & $3 \mathrm{~h} 38 \mathrm{~m}$ & $4 \mathrm{~h} 38 \mathrm{~m}$ \\
\hline
\end{tabular}

then, take advantage of the tracking of multiple GNSS constellations, the GNSS-R instruments have to be equipped with further receiving channels. In the simulated scenarios, a revisit time improvement by $60 \%$ in scenario 3 w.r.t. scenario 1 has been experienced when using a constellation of 32 GNSS-R satellites.

The acquisition of signals coming from different GNSS constellations (as in Scenario 3) leads to a quite surprising result: a lower revisit time can be achieved even with a smaller size of the GNSS-R constellation by tracking more GNSS constellations (see Fig. 5 and compare the red solid line relevant to a constellation of 32 GNSS-R satellites tracking GPS only with the blue dash-dotted one relevant to a constellation of 16 GNSSR satellites tracking GPS, Galileo, GLONASS, and BeiDou-2). However, this is true only when considering a sufficiently high number of receiving channels (six for the simulated scenarios).

Finally, it is interesting to note that all the mentioned solutions can also address a higher homogeneity of the revisit time in the coverage area as confirmed by the first-order statistics of the revisit time shown in Figs. 2-4. Table IV summarizes the revisit time descriptive statistics achieved in the selected scenarios considering a constellation of 32 GNSS-R satellites equipped with 16 receiving channels.

\section{Sea TARget Detection}

In this section, a CFAR sea target detection system from spaceborne GNSS-R imagery is derived and described. Performances are provided by the ROC curves of the algorithm. Gen- 
erally, ship detection algorithms from remote sensing data share a common four-step scheme: land masking, preprocessing, prescreening, and selection [11]. Land masking aims at canceling out the land contributions in the image, in order to focus the algorithm to sea surface only. This step is very important since ship detectors can produce numerous false alarms in land areas [1], [11]. However, this is a standard preliminary stage typically accomplished by applying a land mask derived from shoreline database [1], [38], and we do not focus on this step. The preprocessing step aims at making the detection stages easier. Typically, this stage is carried out by emphasizing the targetto-background ratio by means of targets and/or scene features, e.g., speckle filtering in SAR-based detectors [2], [39]. The preprocessing step can also include calibration and geolocation of the data [1], [11]. As a result, an enhanced image is then derived from the original one. Target candidates are selected in the prescreening stage by hard thresholding the preprocessed image. The threshold can be fixed over all the image or adaptively evaluated (CFAR approach). In the latter case, sea clutter characteristics are accounted for in order to adaptively estimate the local threshold. Threshold has to be designed by taking into account the tradeoff false alarms-detected targets, i.e., the ROC of the detector. If targets are associated with high (low) value of the enhanced image, a high (low) threshold produces low false alarms, but also a low detection rate; vice versa, a low (high) threshold provides a high detection rate and numerous false alarms as well. The last optional selection stage is aimed at reducing the false alarms (or ambiguities as in the SAR case) produced in the prescreening step, thus improving the overall performance of the detector. Target features (size, shape) are commonly accounted for in the final target candidates' selection.

A detailed description of the proposed sea target detector follows.

\section{A. Proposed Sea Target Detection Algorithm}

1) Preprocessing: The backscattering configuration typical of SAR sensors makes sea surfaces backscattered energy weaker than that scattered by complex-shaped man-made objects, like ships [40]. Dihedral structures, typically using in ships, cause a significant amount of energy backscattered to the sensor, while quite flat sea surfaces are characterized by a weak backscattering [5]. This causes ships to be represented as brilliant points in a dark background in the SAR imagery. Similar comments deserve the ship detection from optical data, in which the greater energy reflected by the ship w.r.t. water is related to its materials.

For a complete understanding of the proposed detection approach, the sea target appearance in the GNSS-R DDM deserves some comments in a physical framework. In a forward configuration (as GNSS-R), different scattering mechanisms come into play depending on the actual position of the target within the delay-Doppler domain. If the target is at or sufficiently close to the specular reflection point, the coherent scattering from the flat top plate dominates the radar echo. Very recently, the appearance of coherent scattering phenomena in DDMs of sea-ice sheets has been demonstrated in [29]. In this case, sea targets are

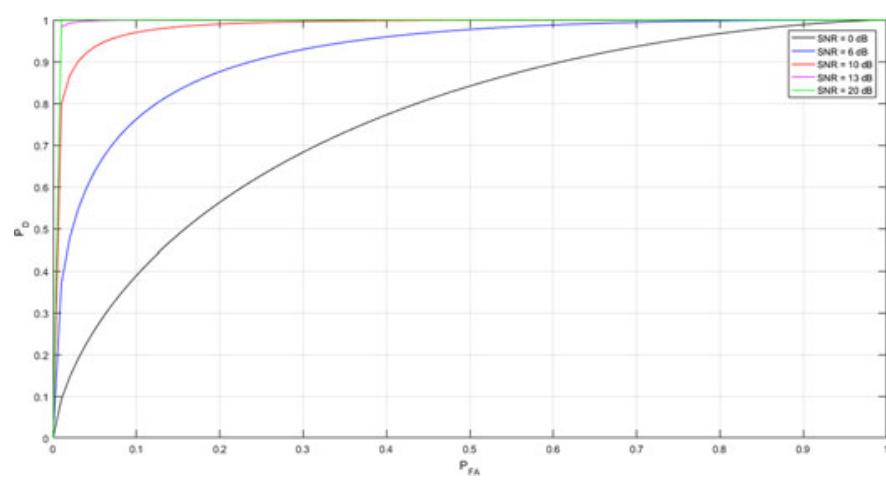

Fig. 6. ROC of the detector. For any fixed $P_{\mathrm{FA}}$, the detection rate increases with the SNR. The tradeoff between the probability of detection and the probability of false alarms is evident: an improvement of the detection rate can be achieved at a cost of an increased probability of false alarms.

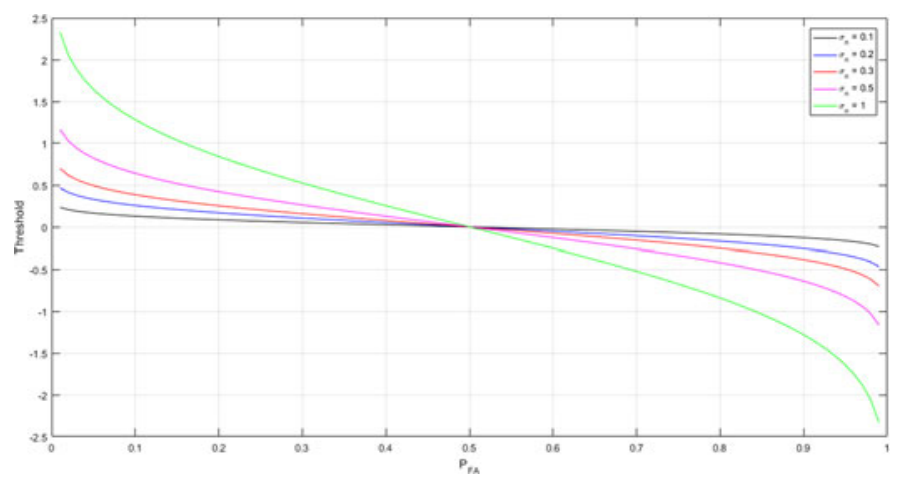

Fig. 7. Threshold versus $P_{\mathrm{FA}}$. For $P_{\mathrm{FA}}$ close to $0\left(P_{\mathrm{FA}}<0.5\right)$, the threshold increases with increasing standard deviation of noise.

TABLE V

U.K. TDS-I PARAMETERS

\begin{tabular}{ll}
\hline \hline Receiver altitude & $640 \mathrm{~km}$ \\
\hline Delay resolution & $244.39 \mathrm{~ns}$ \\
Doppler resolution & $500 \mathrm{~Hz}$ \\
Sampling frequency & $16.37 \mathrm{MHz}$ \\
Coherent integration time & $1 \mathrm{~ms}$ \\
Incoherent integration time & $1 \mathrm{~s}$ \\
\hline
\end{tabular}

expected to appear as very bright feature in the DDM, since they reflect most of the incident electromagnetic energy in the specular direction. On the contrary, if the target is away from the specular point, other scattering phenomena significantly contribute to the energy scattered from the target (edge diffraction, multiple bounce). Furthermore, the complex structure of the considered targets improves the likelihood to observe a greater scattering contribution from the target w.r.t. the sea clutter.

In this case, sea targets are expected to appear as bright features depending on the actual relative position between the transmitter and the target and its orientation.

Therefore, the signal coming from sea targets is expected to be strong enough for target detection purposes. These comments 


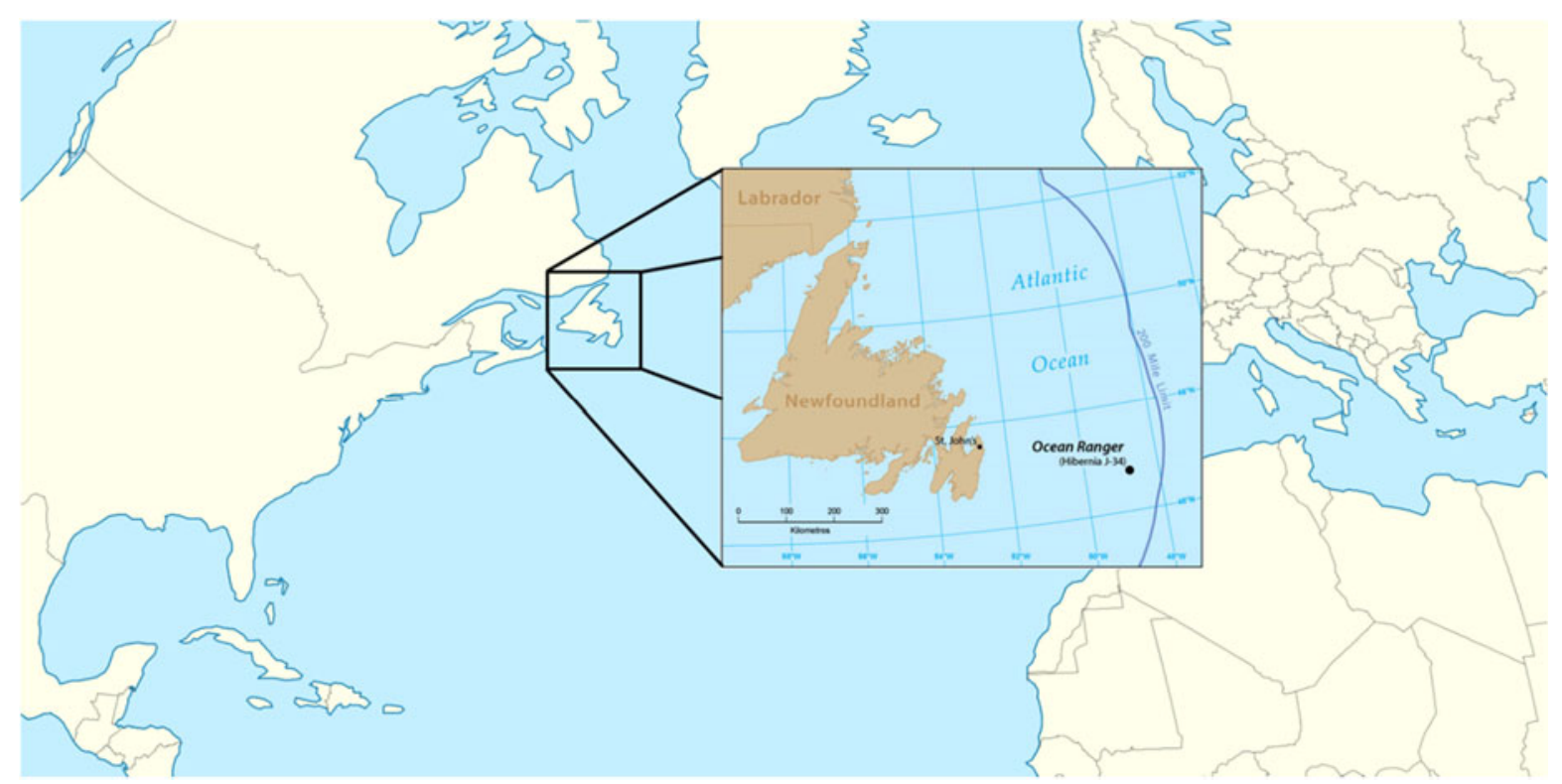

Fig. 8. Hibernia oil rig is situated on the Hibernia oilfield in the North Atlantic Ocean, $315 \mathrm{~km}$ off St. John's, Newfoundland at $46.75{ }^{\circ} \mathrm{N}, 48.78^{\circ} \mathrm{W}$.

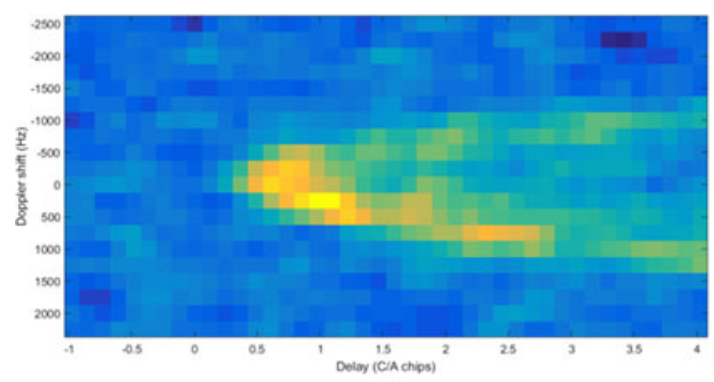

(a)

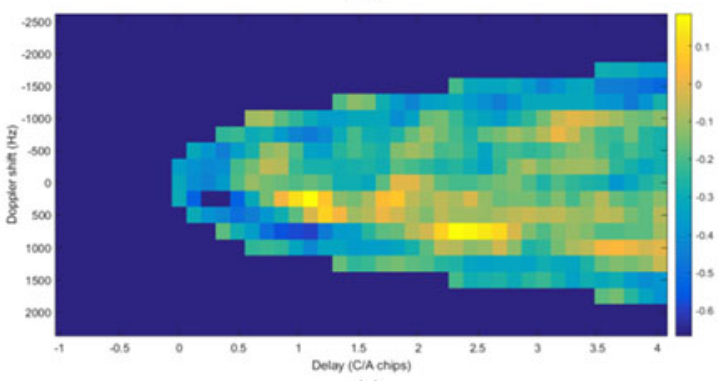

(c)

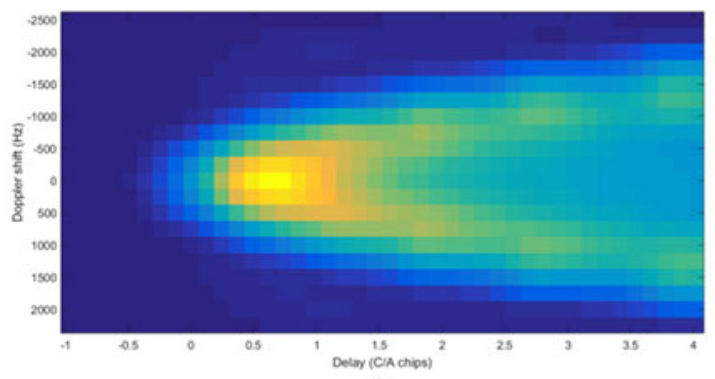

(b)

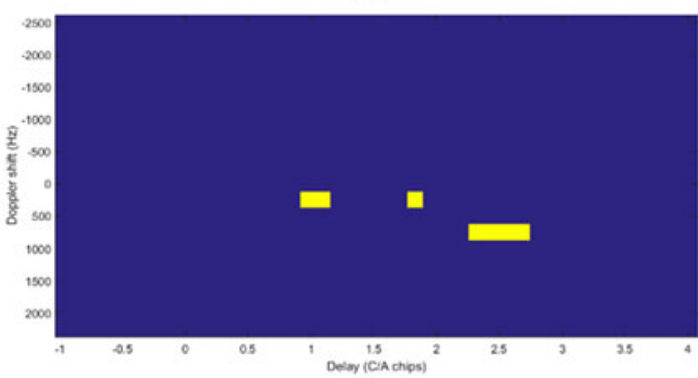

(d)

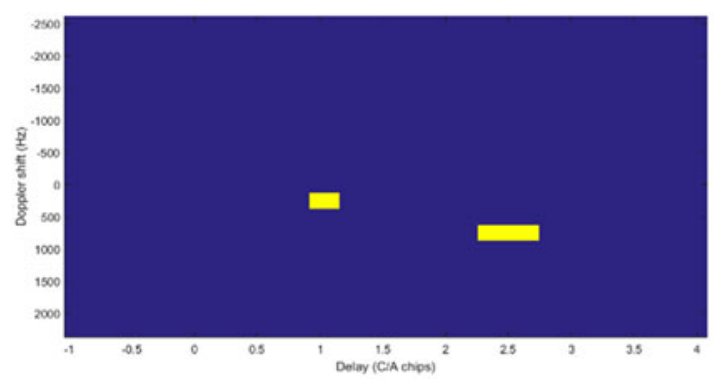

(e)

Fig. 9. (a) TDS-1 DDM acquired on April 1, 2015 at 00:19:36 UTC. Nominal specular point at $46.83{ }^{\circ} \mathrm{N}, 47.53^{\circ} \mathrm{W}$. The visible bright feature is the Hibernia platform situated at about $95 \mathrm{~km}$ off the specular point. (b) Simulated sea clutter contribution. (c) Difference map. (d) Prescreening. (e) Selection. The Hibernia platform is detected on the right. A sea-ice sheet is detected on the left and validated using NSIDC data. 


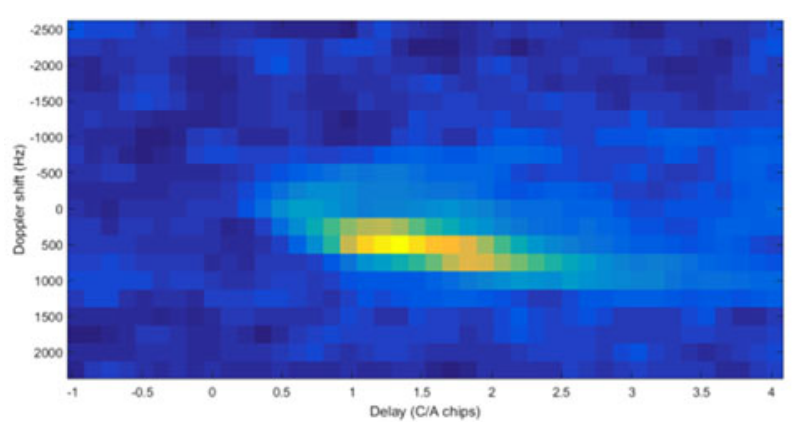

(a)

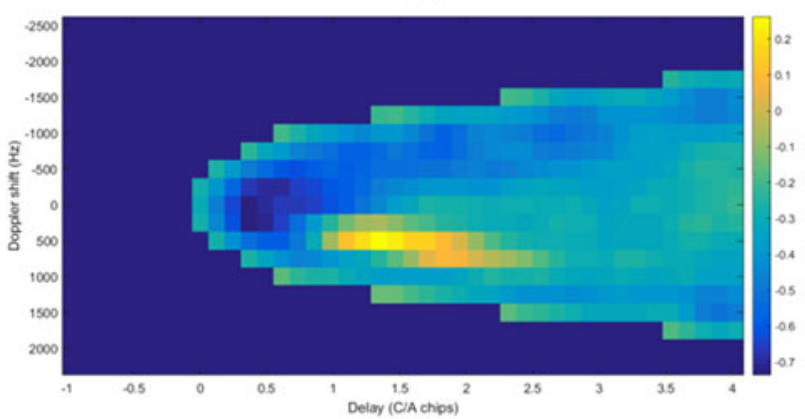

(c)

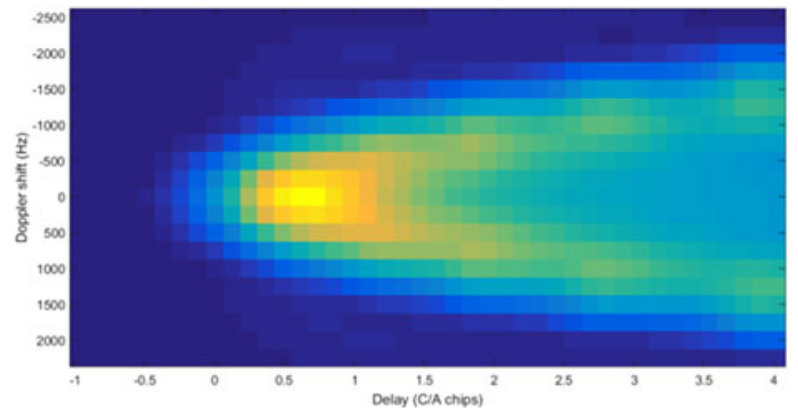

(b)

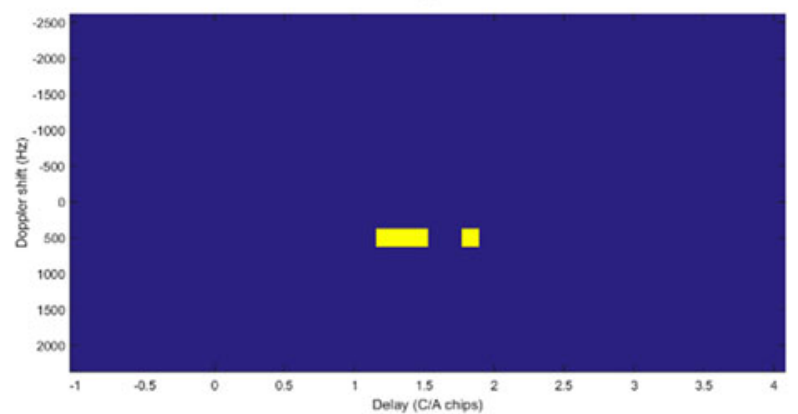

(d)

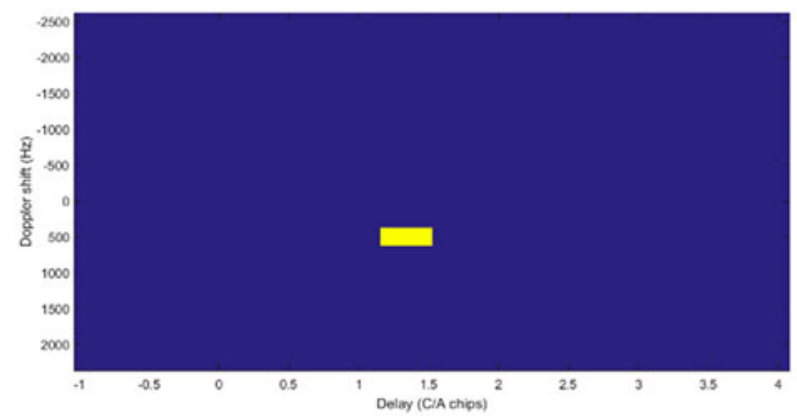

(e)

Fig. 10. (a) TDS-1 DDM acquired on April 1, 2015 at 00:19:49 UTC. Nominal specular point at $47.47^{\circ} \mathrm{N}, 47.84^{\circ} \mathrm{W}$. The visible bright feature is a sea-ice sheet. (b) Simulated sea clutter contribution. (c) Difference map. (d) Prescreening. (e) Selection. The detected target is a sea-ice sheet validated using NSIDC data.

apply also to other complex structures over the sea surface, such as oil and gas platforms.

It is finally noteworthy that the bistatic configuration of GNSS-R systems is more favorable to the detection of stealth ships w.r.t. monostatic radar systems, such as SAR. Stealth ships are typically designed to be "invisible" to monostatic radars, which represent the most common system in military applications. Hence, the stealth design process is typically aimed at lowering as much as possible the radar cross section in the backscattering direction, so that the ship is "invisible" in a monostatic configuration. However, the direction in which most of the energy is diverted is not easy controlled, since it depends on the actual relative position between the transmitter and the target and its orientation. This makes more likely to measure a higher signal-to-clutter ratio in a bistatic configuration (such as GNSS-R) rather than monostatic (such as SAR) [41].

The bistatic configuration typical of GNSS-R systems makes the sea clutter a nonnegligible contribution in the DDMs, so that a clutter compensation step is a desirable step to enhance the presence of potential targets over the sea. In this paper, the preprocessing step consists of a clutter estimation and cancelation stage by means of DDM simulation. The main aim of this step is to cancel out the sea clutter contribution within the glistening zone in order to bring the target out of the background.

In this paper, the $\mathrm{P}^{2}$ EPS (PAU/PARIS End to end Performance Simulator) tool has been used for simulation purposes [42], while the simulated DDM has been obtained by leastsquares fitting (LSF) with the measured one. The LSF step is performed assuming an unknown elevation angle, i.e., angle $\gamma$ in Fig. 3 in [43]. Although there are other unknown parameters, such as $\alpha_{R}$ and $\alpha_{\mathrm{T}}$ as defined in Fig. 3 in [43], we disregarded their role since a minor influence w.r.t. $\gamma$ was shown in the LSF step and performed the LSF stage w.r.t. $\gamma$ only. This choice leads to a simplified LSF step, since only one parameter has to be tuned. To ensure an unbiased clutter compensation, the measured DDM is compensated for the thermal noise power as well. The noise power is estimated as the mean value of the pixels in the forbidden zone, i.e., the area of the Delay-Doppler domain not corresponding to any physical area. Indeed, in this 


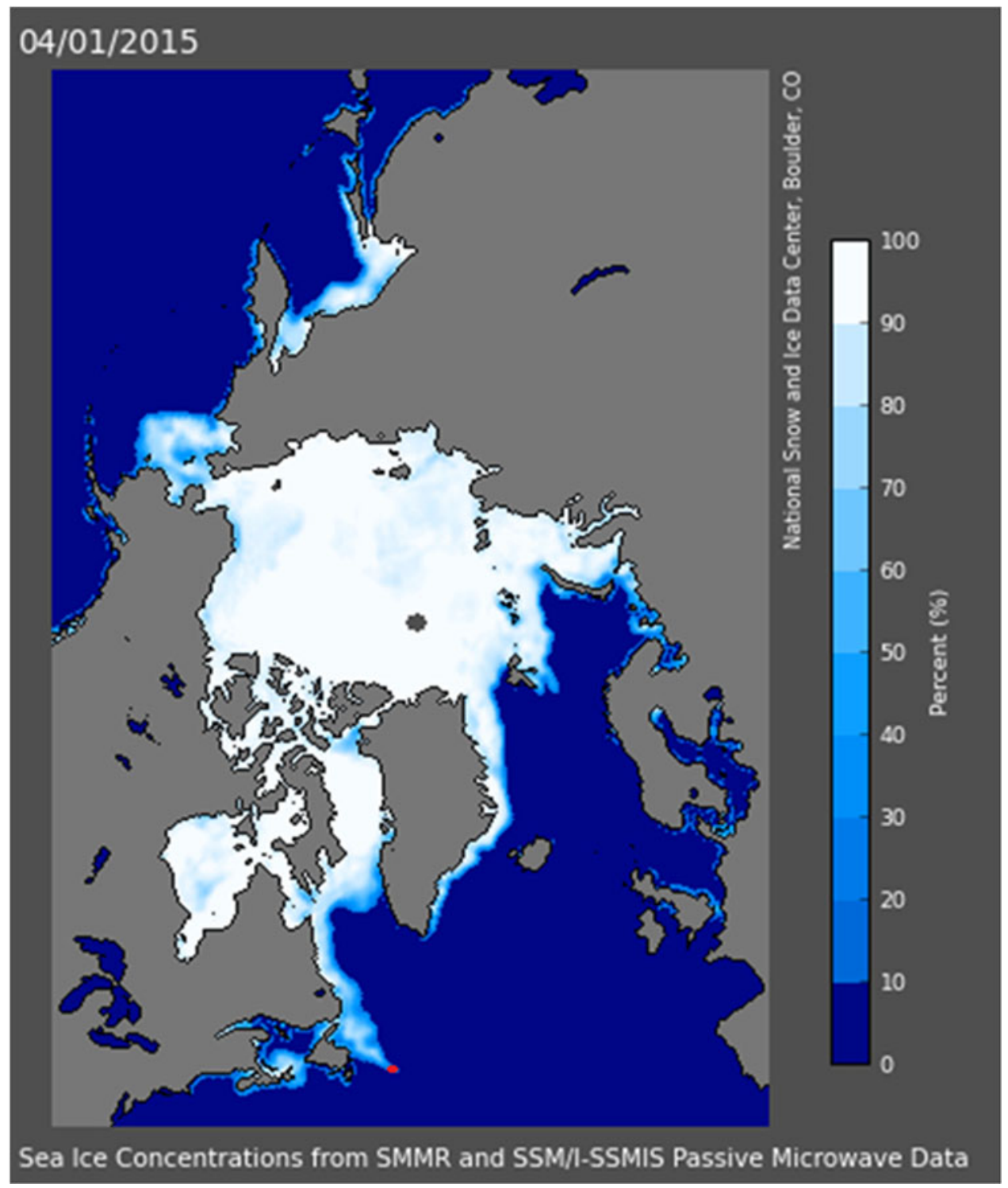

Fig. 11. Image representation of the NSIDC sea-ice concentrations data used for validation of sea-ice sheets detection. The red point indicates the target detected by the algorithm.

area, no signal coming from neither sea nor targets is measured, and the thermal noise is the only contribution. Noise powercompensated DDM and the simulated one are then normalized to their peak; the preprocessed image is then defined as the pixelwise difference between the normalized actual and simulated DDMs. The output of this stage is a difference map of the glistening zone, in which the horseshoe pattern, i.e., sea clutter, typical of spaceborne DDM over the sea, has been canceled out.

2) Prescreening: In the prescreening stage, bright features in the difference map are associated with possible targets; therefore, a hard-thresholding is applied to the difference map to provide the target candidates. In order to account for clutter inhomogeneity and thermal noise, a CFAR approach is used at this stage. The pixel under test is then compared with a threshold adaptively evaluated by means of a sliding-moving window as explained in more detail in Section III-B.
3) Selection: In the selection stage, isolated targets, i.e., single-pixel targets, are supposed to be likely false alarms caused by noise. Hence, isolated bright pixels are likely to be spike noise randomly exceeding the local threshold. In support of this assumption, the power spreading effect caused by the point spread function (PSF) comes into play. In the presence of large features on the sea surface (large ships and sea-ice sheets), the PSF spreads the received power on neighboring pixels, thus increasing their correlation. In the presence of noise only, it is more unlikely to observe neighboring pixels exceeding the threshold, and then, single-pixel targets appear.

Isolated, i.e., single-pixel, targets are removed from the prescreened candidates map by means of a morphological operation. False alarms caused by small-to-medium isles, airplanes, speckle noise, etc., are still possible. 


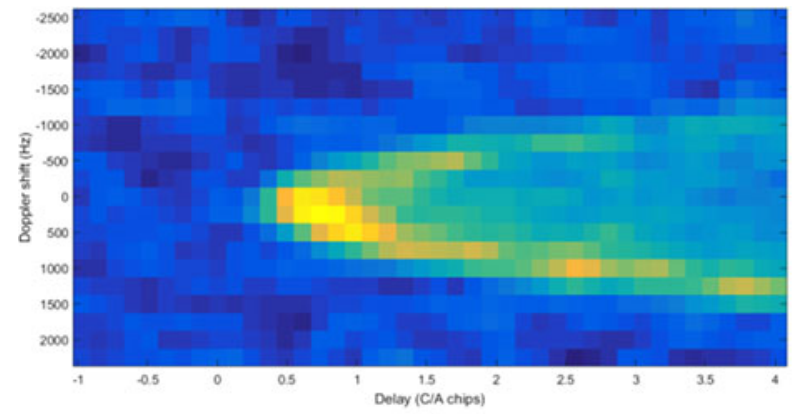

(a)

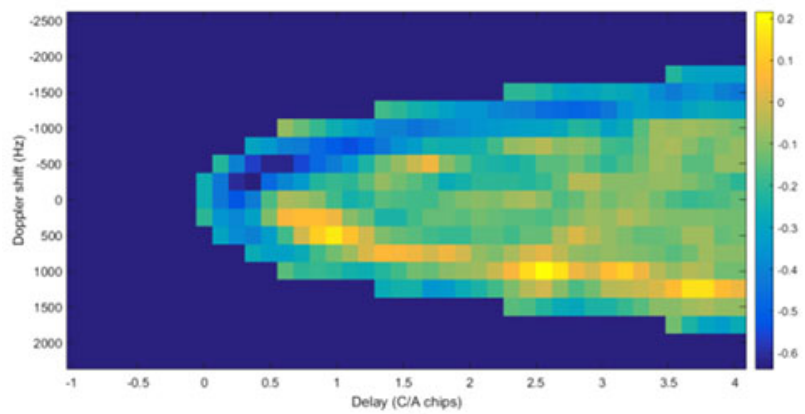

(c)

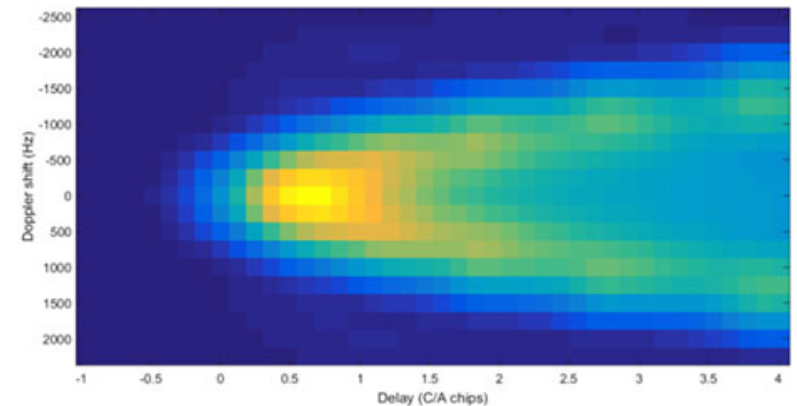

(b)

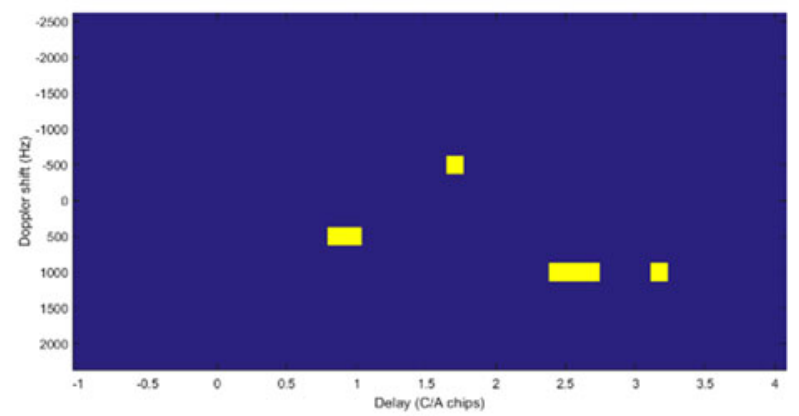

(d)

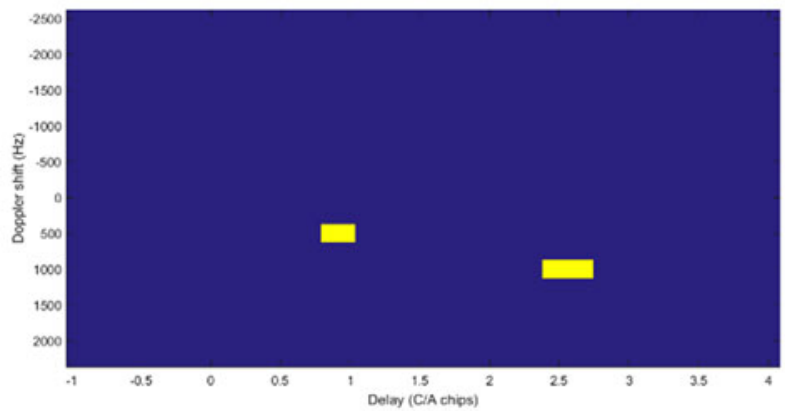

(e)

Fig. 12. (a) TDS-1 DDM acquired on February 28, 2015 at 16:18:32 UTC. Nominal specular point at $27.45^{\circ} \mathrm{N}, 89.45^{\circ} \mathrm{W}$. (b) Simulated sea clutter contribution. (c) Difference map. (d) Prescreening. (e) Selection.

4) Geolocation: In the geolocation stage, the geographic locations of the detected targets in the delay-Doppler domain are identified. In order to find the geographic coordinates of the detected targets, the observation geometry should be reconstructed in the geographic reference frame, i.e., the positions and velocities of transmitter, receiver, and the specular reflection points are needed. Those data are available from the auxiliary of spaceborne GNSS-R mission, e.g., TDS-1 case from MERRByS website (http://www.merrbys.co.uk/).

Once the observation geometry is reconstructed, the positions in the delay-Doppler domain can be linked to the position in the geographic coordinate system.

One consideration in the geolocation process is ambiguity of bistatic reflection geometry. In fact, a single position in the delay-Doppler domain corresponds to two different points in the spatial domain (see [44, Fig. 1]). It means that, within the proposed approach, the detected targets from DDM can be assigned to two different geographic locations. To solve the ambiguity, the multibeam method has been proposed [23][25]. However, the multibeam (or beam steering) method is not suitable for small (nano-, cube-) satellite platforms because of the system complexity and the power, size, and weight constraints.

In the fixed single-beam case, the multiple overpasses can be used. A false location can be filtered out from the DDMs acquired from multiple overpasses on the target area. This multishot and multipass process also increases the accuracy of geolocation. For the other method, the auxiliary images from the other type of sensors can be used to overcome the ambiguity problem.

\section{B. Performance Assessment}

The performance of the proposed algorithm is theoretically assessed by evaluating the ROCs of the detector. These curves allow a fair performance assessment since they are not based on specific thresholds values. To this aim, the following hypothesis testing is considered for any pixel inside the glistening zone:

$$
\left\{\begin{array}{l}
H_{1}: y=s+c+n \\
H_{0}: y=c+n
\end{array}\right.
$$




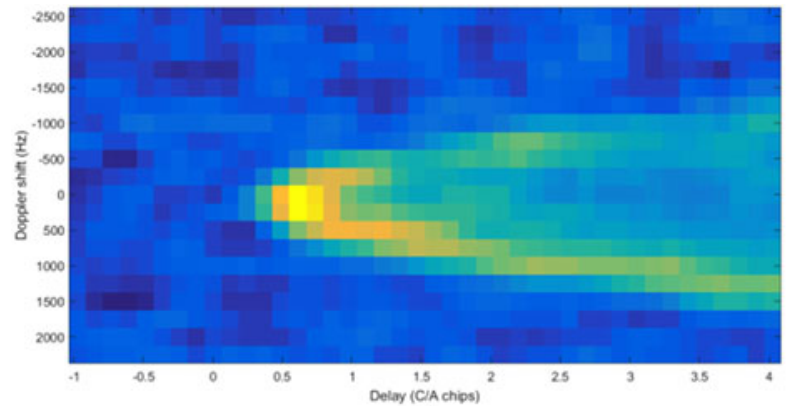

(a)

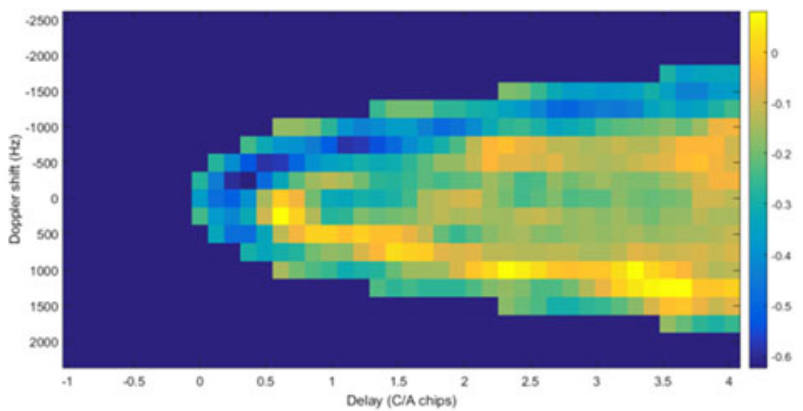

(c)

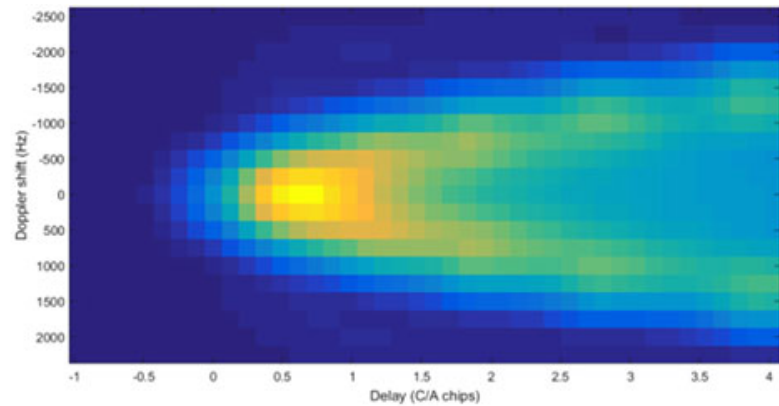

(b)

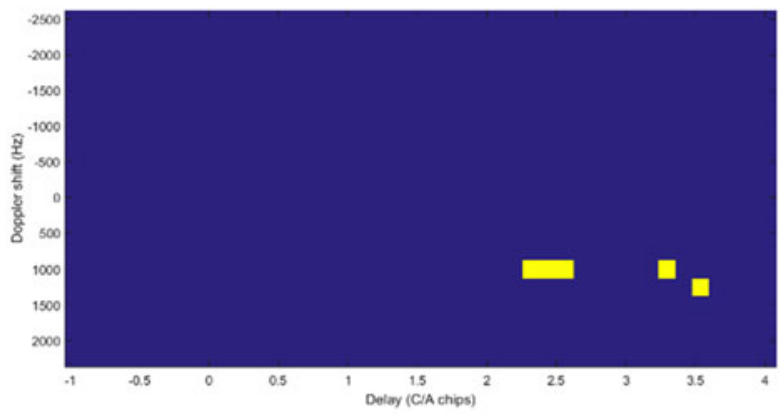

(d)

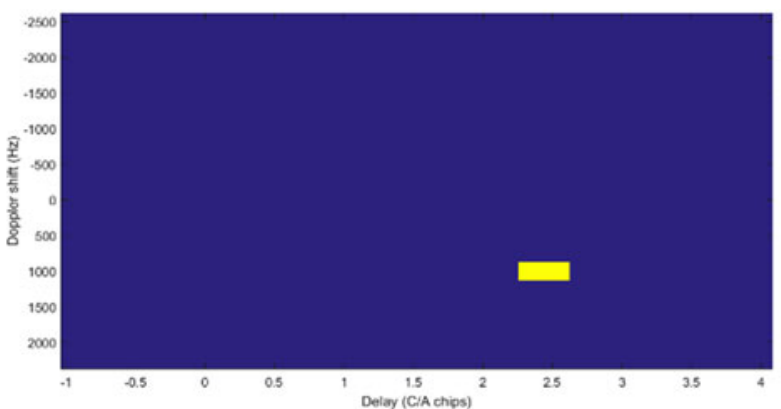

(e)

Fig. 13. (a) TDS-1 DDM acquired on February 28, 2015 at 16:18:33 UTC. Nominal specular point at $27.40^{\circ}$ N, 89.47 W. (b) Simulated sea clutter contribution. (c) Difference map. (d) Prescreening. (e) Selection.

where $y$ is the pixel intensity of the incoherently averaged DDM, $s$ is the target signal intensity, $c$ stands for the sea clutter, and $n$ represents the thermal noise. The physical-based considerations in Section III-A-1 justify the hypothesis in (1), in which the observed power in the pixel (in the delay-Doppler domain) containing a ship will exceed that from the sea clutter. Assuming that the thermal noise is a normally distributed random variable in the coherently-averaged DDM, the noise term $n$ in (1) follows a chi-squared distribution in the incoherently-averaged DDM, i.e., $n \sim \chi^{2}(k), k$ being the number of incoherently averaged DDMs, i.e., the ratio between the incoherent and coherent integration times, respectively; typical GNSS-R systems, such as TDS-1, CYGNSS, perform a 1-ms coherent acquisition and 1-s incoherent averaging [46], [47], so that in practical cases, $k \gg 1$ can be assumed. Recalling the central limit theorem, a normally distribution can be assigned to the noise term in (1), i.e., $n \sim N\left(\mu_{n}, \sigma_{n}\right)$.

The noise power compensation is as

$$
y^{\prime}=y-\mu_{n}
$$

and the hypothesis testing becomes

$$
\left\{\begin{array}{l}
H_{1}: y^{\prime}=s+c+n \\
H_{0}: y^{\prime}=c+n
\end{array}, n \sim N\left(0, \sigma_{n}\right)\right.
$$

the thermal noise being a zero-mean normally distributed random variable at this stage.

The preprocessing step is aimed at removing the sea clutter contribution in the hypothesis testing in (3), i.e., the new observable is the difference map $d=y^{\prime}-\hat{c}$, where $\hat{c}$ is the estimated sea clutter. To simplify the mathematical derivation of the ROCs, we suppose a perfect sea clutter suppression, i.e., $\hat{c}=c$; consequently, the hypothesis testing reads as

$$
\left\{\begin{array}{l}
H_{1}: d=s+n \\
H_{0}: d=n
\end{array}, n \sim N\left(0, \sigma_{n}\right) .\right.
$$

In the prescreening stage, a CFAR detector is applied, i.e., an adaptive threshold $T$ is estimated in the neighboring of the pixel under test, once assigned a probability of false alarm $P_{\mathrm{FA}}$. 


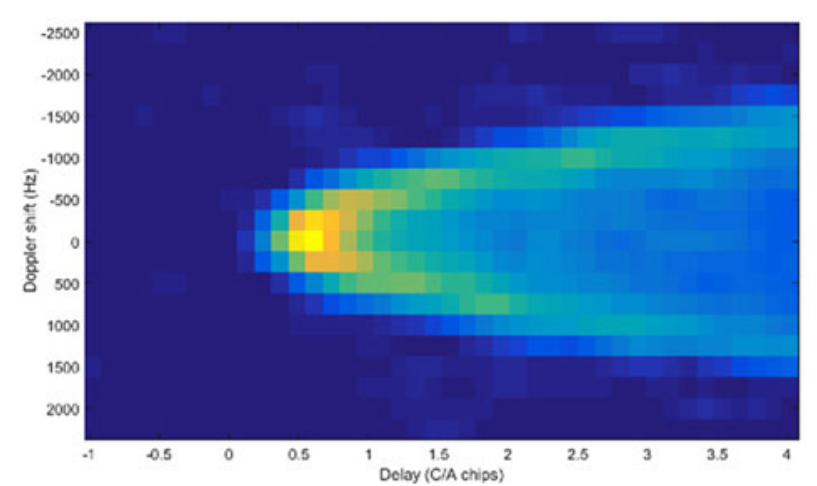

(a)

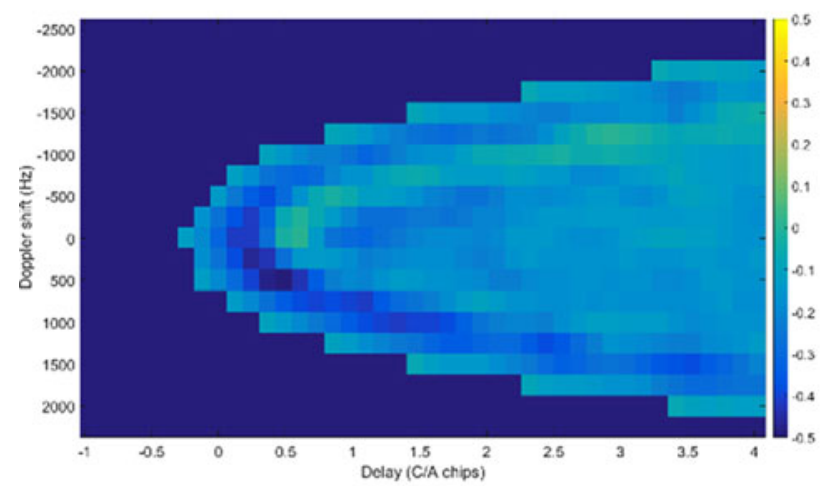

(c)

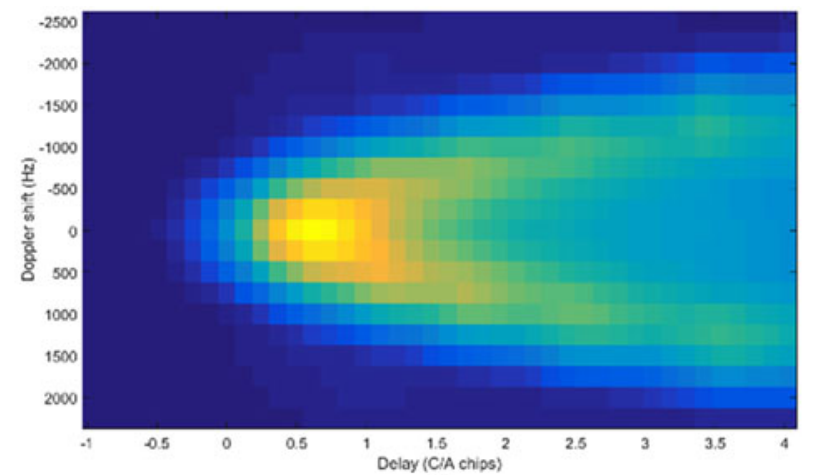

(b)

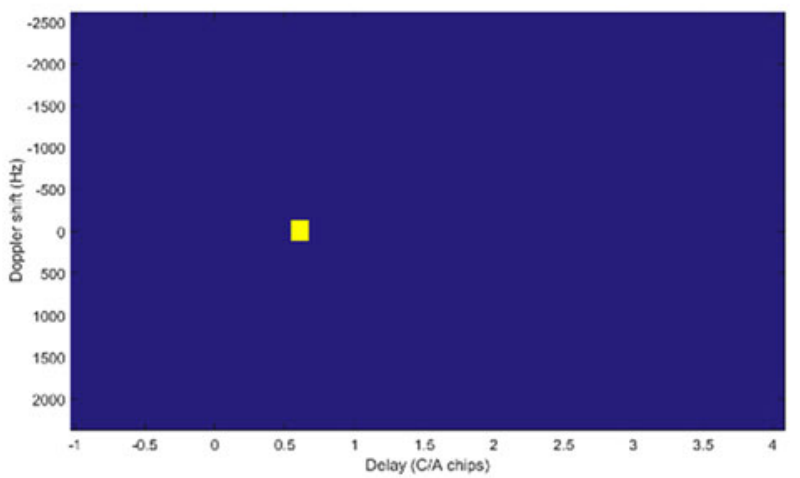

(d)

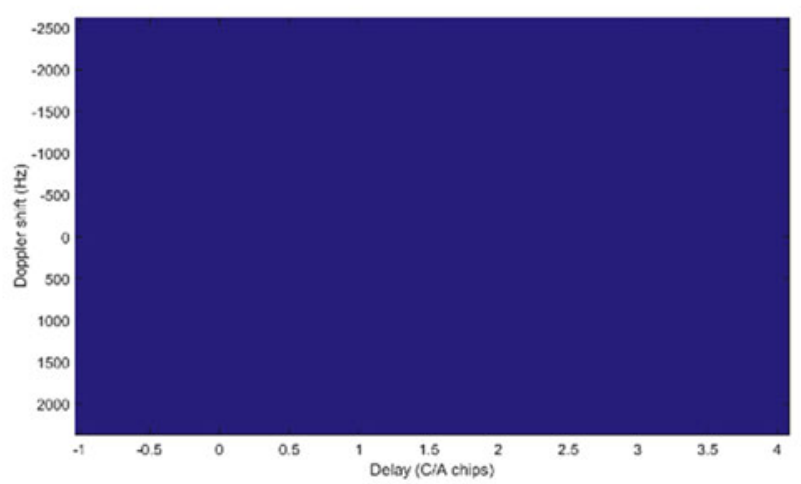

(e)

Fig. 14. (a) TDS-1 DDM acquired on January 27, 2015 at 02:58:22 UTC. Nominal specular point at $-54.41^{\circ} \mathrm{N}, 90.98^{\circ} \mathrm{E}$. (b) Simulated sea clutter contribution. (c) Difference map. (d) Prescreening. (e) Selection.

The probability $P_{\mathrm{FA}}$ can be written as follows:

$$
P_{\mathrm{FA}}=\operatorname{Pr}\left(d>T \mid H_{0}\right)=Q\left(\frac{T}{\sigma_{n}}\right)
$$

where $Q(\cdot)$ denotes the Q-function defined as

$$
Q(x)=\frac{1}{\sqrt{2 \pi}} \int_{x}^{+\infty} \exp \left(-\frac{u^{2}}{2}\right) d u .
$$

Consequently, the local threshold reads as

$$
T=\sigma_{n} Q^{-1}\left(P_{\mathrm{FA}}\right)
$$

where $Q^{-1}(\cdot)$ stands for the inverse Q-function. In order to compute the local threshold in the prescreening step, the noise standard deviation needs to be estimated. In order to account for different error source in the difference map, such as residual sea clutter, speckle noise, imperfect coregistration, etc., the $\sigma_{n}$ parameter is adaptively evaluated through the image, i.e., a CFAR approach is applied. In particular, $\sigma_{n}$ is estimated within a $K \times K$ window centered in the pixel under test. Once $\sigma_{n}$ has been estimated, the local threshold is then evaluated from (6). However, the $\sigma_{n}$ estimation deserves a specific comment. To take into account potential extended targets and the spreading effects of the PSF in the delay-Doppler domain, a $L \times L$ guard window $(L<K)$ centered in the pixel under test is considered and not used for the $\sigma_{n}$ estimation. Therefore, the guard window size influences the size of detectable targets, and its value has to be chosen as the maximum between one and the ratio between the target maximum size and the mean spatial resolution of the GNSS-R system. To avoid missing targets due to the PSF energy 


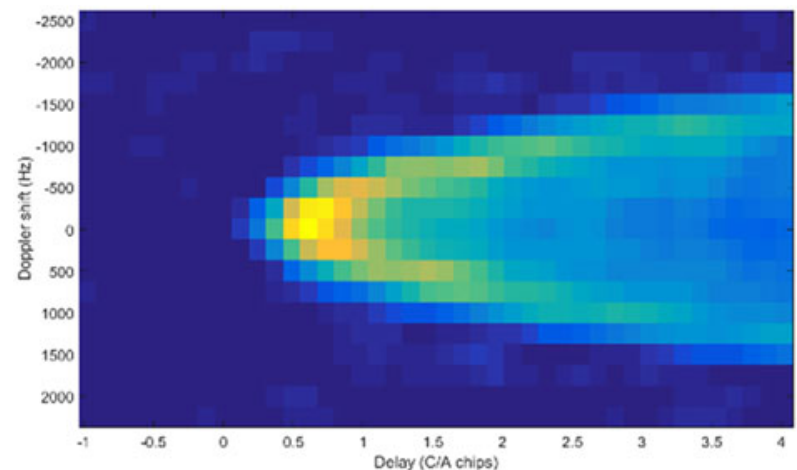

(a)

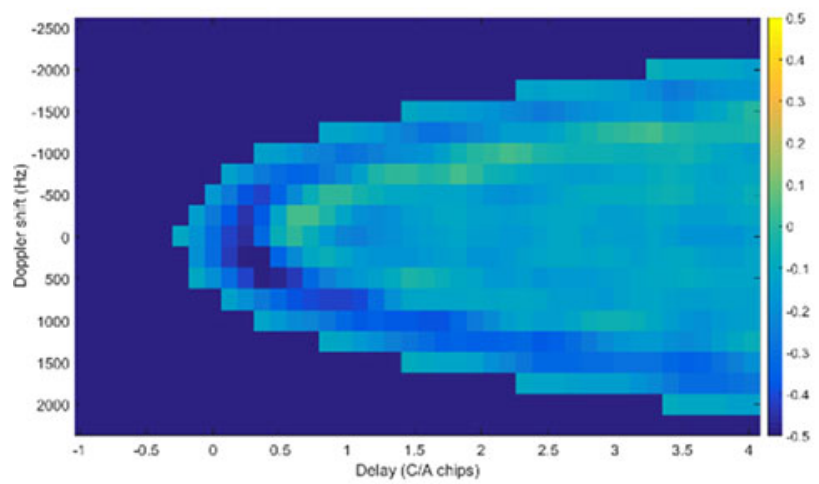

(c)

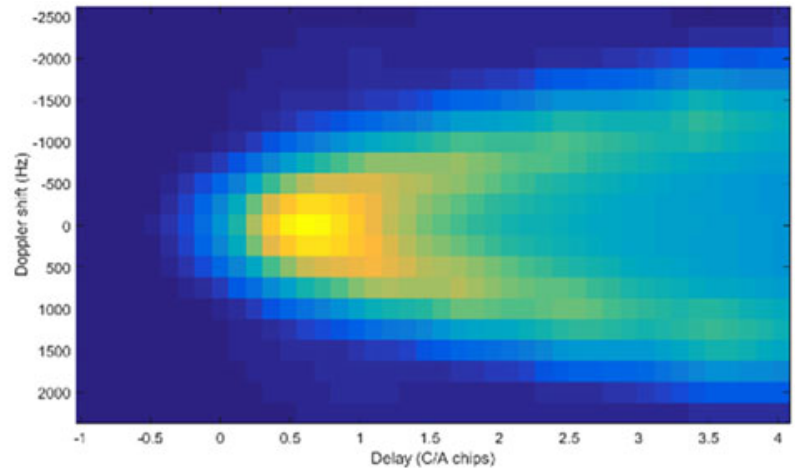

(b)

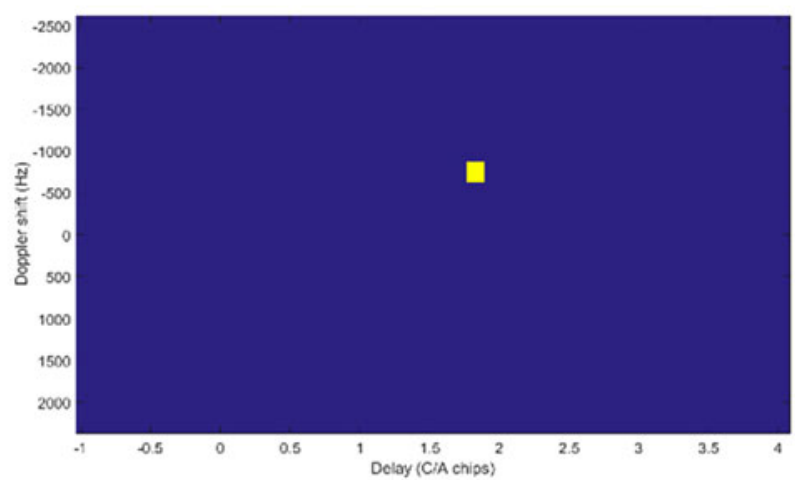

(d)

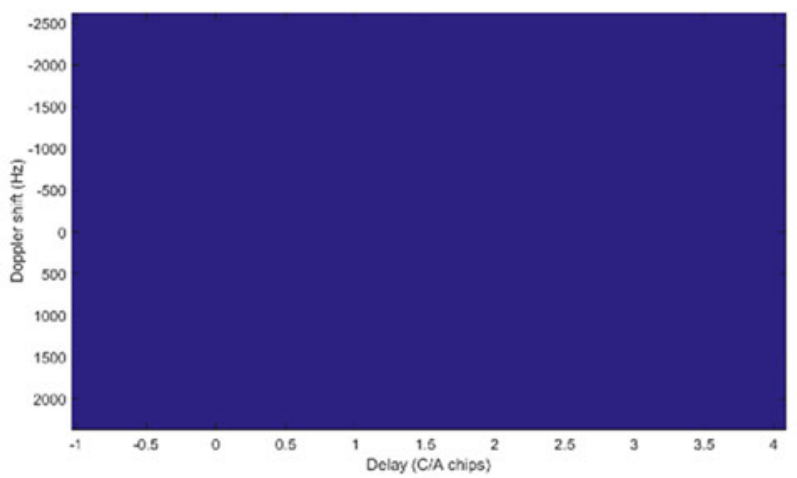

(e)

Fig. 15. (a) TDS-1 DDM acquired on January 27,2015 at 02:58:29 UTC. Nominal specular point at $-54.84{ }^{\circ}$ N, $90.78^{\circ}$ E. (b) Simulated sea clutter contribution. (c) Difference map. (d) Prescreening. (e) Selection.

spreading effect, this value has to be incremented in both delay and Doppler directions of a number of pixels equal to the ratio between the length of the PSF and the delay-Doppler resolutions of the sensor, where the PSF lengths in delay and Doppler are $\tau_{c}\left(1+\frac{\tau_{c}}{T_{i}}\right)$ and $1 / T_{i}$, respectively, $\tau_{c}$ and $T_{i}$ being the chip length and integration time, respectively.

The probability of detection $P_{D}$ is by definition:

$$
P_{D}=\operatorname{Pr}\left(d>T \mid H_{1}\right)
$$

and can be related to the probability of false alarms and the signal-to-noise ratio (SNR) to provide the ROC curve of the detector. For the proposed detector, the ROC reads as

$$
P_{D}=Q\left(Q^{-1}\left(P_{\mathrm{FA}}\right)-\mathrm{SNR}\right)
$$

where $\mathrm{SNR}=s / \sigma_{n}$ is the SNR. The ROC curve of the detector is shown in Fig. 6 for different values of the SNR and represents the performance of the proposed detector as a function of the probability of false alarms. The tradeoff between the probability of detection and the probability of false alarms is evident: an improvement of the detection rate can be achieved at a cost of an increased probability of false alarms. In Fig. 7, the threshold is shown as a function of the $P_{\mathrm{FA}}$ for different values of $\sigma_{n}$ as stated in (6).

\section{EXPERIMENTAL RESULTS}

In this section, the proposed ship detection algorithm is tested on actual U. K. TDS-1 DDMs, and some preliminary results are shown. Orbital and sensor parameters are listed in Table V. In the 


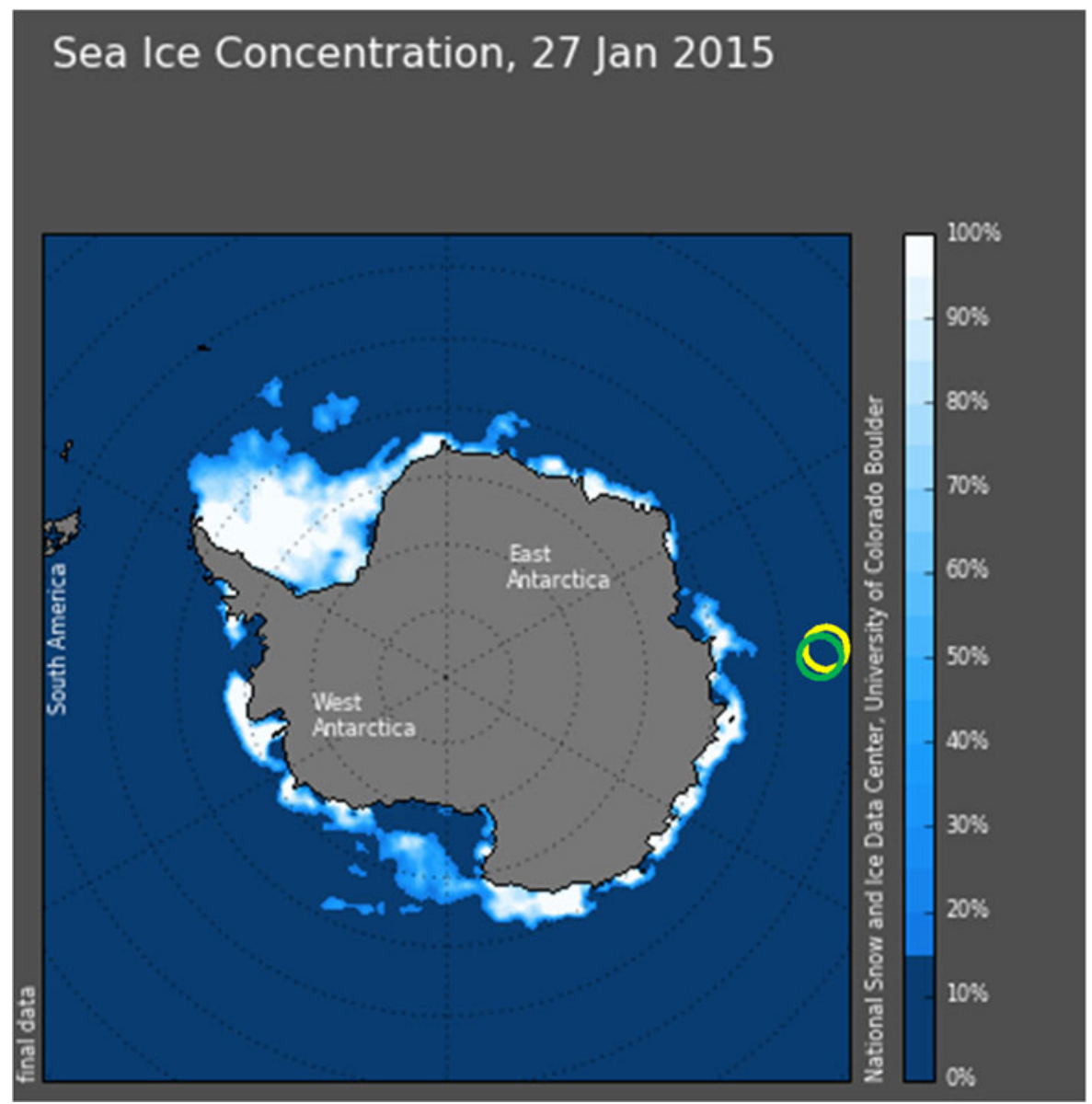

Fig. 16. Image representation of the NSIDC Antarctica sea-ice concentrations on January 27, 2015, relevant to the target-free study case. The yellow and green circles indicate the glistening zones corresponding to the study cases in Figs. 14 and 15, respectively.

preprocessing step, the LSF is performed on the elevation angle as defined in [43] and in the $\mathrm{P}^{2} \mathrm{ESP}$ simulation tool. Even though the elevation angle is related to the incidence angle as defined in [43], the incidence angle provided by MeRRByS in the TDS-1 data was not consistent with the scattering reference frame defined in [43]. Remaining orbital and sensor parameters, as well as wind speed, are taken from ancillary data. Two relevant comments are now in order.

First, it is noteworthy that, at least in principle, the proposed algorithm can be applied even if such a priori sea state information (wind speed) is not available. In this case, the LSF step is performed on a 2-D space (wind speed-elevation angle) in order to estimate the reference sea clutter DDM. The a priori lack of information lack requires an increased execution time since a 2-D instead of 1-D LSF is required.

Second, the LSF step is performed assuming a homogenous wind field over the entire glistening zone. Inhomogeneities in wind speed are not accounted for in the proposed algorithm and can lead to false alarms in the selected targets map, although, fortunately, wind fields do not change significantly within the size of the glistening zone. For instance, a low wind speed field close to the specular point may cause false alarms in the detection step, since, in the calmer sea region, a stronger return is experienced. In any case, methods accounting for inhomogeneities in the wind field map are now available [48].

The difficulties to find a proper ground truth regarding historical ship position and routes free of charge, together with the difficulty to visually assess the presence of ship targets within GNSS-R observables, have been partially compensated by applying and testing the detector on actual DDM data acquired close to offshore oil and gas platforms. Such platforms are static man-made objects, whose location is available for free in many cases. Therefore, they represent suitable sea targets to test the proposed detector in open seas scenarios. The oil platform considered in this paper is the Hibernia Platform (see Fig. 8), situated on the Hibernia oilfield in the North Atlantic Ocean, $315 \mathrm{~km}$ off St. John's, Newfoundland, at $46.75^{\circ} \mathrm{N}, 48.78^{\circ} \mathrm{W}$ [49]. A probability of false alarms equal to 0.01 is considered. The first TDS-1 dataset used to test the algorithm, shown in Fig. 9(a), was acquired on April 1, 2015, at 00:19:36 UTC; the nominal specular point is at $46.83^{\circ} \mathrm{N}, 47.53^{\circ} \mathrm{W}$. The Hibernia platform is $95 \mathrm{~km}$ off the specular point, and it is visible as an extended bright feature in the measured DDM due to the PSF. The preprocessing step [see Fig. 9(c)] suppresses the sea clutter estimated in the simulated DDM in Fig. 9(b); the Hibernia oil rig emerges from sea clutter and appears like a quite bright ex- 
tended feature in Fig. 9(c) due to the PSF. In the prescreening stage, the oil rig is correctly detected; other two sea targets are detected as well: a single-pixel target and an extended target. The isolated target is rejected as noise in the successive selection stage [see Fig. 9(e)], as explained in Section III-A 3, while the extended target is relevant to a region with a sea-ice concentration greater than zero, as reported in the NSIDC sea-ice concentration map relevant to the same day [50] (see Fig. 11). Detection of the same region is shown in Fig. 10. In both cases, a sea-ice concentration of more than $20 \%$ has been obtained in correspondence of the detected targets using the NSIDC map. The actual DDM shown in Fig. 10(a) was acquired on April 1, 2015 at 00:19:49 UTC, i.e., $13 \mathrm{~s}$ after the previous actual case. The nominal specular is at $47.47^{\circ} \mathrm{N}, 47.84^{\circ} \mathrm{W}$, and the sea-ice sheet is visible as an extended target at about $1.34 \mathrm{C} / \mathrm{A}$ chips and $500 \mathrm{~Hz}$ in the delay-Doppler domain in both the measured DDM and the difference map [see Fig. 10(c)]. The prescreening stage correctly detects the target [see Fig. 10(d)]; an isolated target is removed in the selection stage, since it is likely to be due to noise, as explained in Section III-A 3 [see Fig. 10(f)].

In the second study case, the proposed sea target detector is applied to two consecutive DDMs acquired in the Gulf of Mexico (U.S.) on February 28, 2015 at 16:18:32 UTC [see Fig. 12(a)] and 16:18:33 UTC [see Fig. 13(a)], respectively. The nominal specular points, at $27.45^{\circ} \mathrm{N}, 89.45^{\circ} \mathrm{W}$ and $27.40^{\circ} \mathrm{N}, 89.47^{\circ} \mathrm{W}$, respectively, are about $180 \mathrm{~km}$ off the New Orleans, LA, coast, so that no significant contribution from land areas are expected in the considered dataset. The estimated sea clutter contribution in the delay-Doppler domain is shown in Figs. 12(b) and 13(b) and subtracted from the measured DDMs in the difference maps shown in Figs. 12(c) and 13(c). The prescreening stage detects four [see Fig. 12(d)] and three [see Fig. 13(d)] target candidates, respectively; two of which rejected in the selection stage as shown in the selected target maps in Figs. 12(e) and 13(e). Gulf of Mexico is a major source of oil and gas in the United States [51]; indeed, the area is occupied by more than 200 of oil and gas platforms [52], and a huge maritime traffic takes place every day in the area [6]. Consequently, the detected targets are presumably actual sea targets (ships, oil platforms). The detection of a target on both DDMs in quite the same position reinforces this chance.

For a comprehensive analysis, the last study case aims at analyzing the detector performance in target-free scenarios. The selected region is located in the southern regions of Indian Ocean, in which neither sea traffic nor sea-ice sheets are revealed (see the black circled area in Fig. 1 and the Antarctica sea-ice concentration in Fig. 16). The two selected TDS-1 DDMs were acquired on January 27, 2015 at 02:58:22 UTC [see Fig. 14(a)], and on January 27, 2015 at 02:58:29 UTC [see Fig. 15(a)]. The nominal specular points are located at $-54.41{ }^{\circ} \mathrm{N}, 90.98^{\circ} \mathrm{E}$ and $-54.84{ }^{\circ} \mathrm{N}, 90.78^{\circ} \mathrm{E}$, respectively. The simulated targetfree DDMs are shown in Figs. 14(b) and 15(b), respectively. In both cases, no visible features appear in the difference map [see Figs. 14(d) and 15(d)], which exhibits a homogeneous pattern. In both cases, a false alarm single-pixel target is detected in the prescreening stage and removed in the subsequent selection stage. Consequently, no targets were identified in this selected dataset.

\section{CONCLUSION}

In this study, a feasibility study of real-time sea target detection from GNSS-R observables has been assessed. Owing to low cost and low size, small-satellite GNSS-R constellations can be developed at a much lower cost compared with competitive remote sensing techniques, giving the chance for a near real-time worldwide sea traffic monitoring and control. An experimental study of the revisit time provided by GNSS-R constellations has been conducted by means of mission simulations and analysis. This experimental study aims at providing some practical guidelines for the mission design, by analyzing the most relevant adjustable parameters influencing the time resolution and providing synthetic numerical results on realistic test missions. Three different scenarios have been defined and simulated in order to evaluate some first-order statistics of the revisit time, namely average, median, and standard deviation. It has been shown that, even if single GNSS-R systems can provide time resolution similar to other remote sensing technologies, such as SAR and optical systems, the strength of GNSS-R systems lies in the actual chance to group them in cooperative formations, which can offer revisit time as low as $2 \mathrm{~h}$ or even lower. The actual revisit time depends upon numerous parameters, such as the number of satellites, the number of parallel tracking channels, global coverage, and the GNSS stations tracked. Increasing the number of tracked signals allows for a limited improvement in the revisit time, this plateau lowering with increasing GNSS transmitters that can potentially be tracked. Increasing the constellation size represents the only method to reach an arbitrarily low revisit time. This potentiality paves the way to a spread array of Earth observation applications with strict time requirements. One of the most interesting for its key role in worldwide economic and social activities, i.e., sea target detection, has been explored, theoretically assessed, and also experimentally validated using U.K. TDS-1 data. The target detector consists of four processing stages: in the preprocessing stage, the sea clutter contribution is estimated in the delay-Doppler domain by means of a least-squares approach via the $\mathrm{P}^{2} \mathrm{ESP}$ simulator; the sea clutter term is then subtracted from the actual DDM to form the target enhanced image. A CFAR thresholding is applied to provide the target candidates map in the prescreening step. The selection stage reduces the false alarms rate by rejecting isolated detected targets. The geolocation step identifies the geographical coordinates of the detected targets, while no solutions are implemented in this work to deal with the spatial ambiguity of the target location. The ROC curves of the detector have been derived for the performance assessment. The validation of the proposed algorithm using actual GNSS-R imagery represents another contribution of this paper. The difficulties to exploit a proper ground-truth information about location of ships has been circumvented by testing the detector on an oil platform owing to the exploitation of ground truth about its location at no cost. Two U.K. TDS-1 DDMs acquired on the 
North Atlantic have been used to assess the capability of the algorithm to detect the Hibernia oil platform. Another case study has been defined in the Gulf of Mexico, due to its key role in worldwide sea trades. Finally, a target-free scenario has been analyzed using DDMs acquired in the Southern regions of the Indian Ocean, in which neither sea traffic nor sea-ice sheets were present. This feasibility study is essential for understanding the role of upcoming GNSS-R constellations in the framework of sea target detection and feature mapping and could promote innovative solutions in the integration of remotely sensed data acquired by different sources. A valid guideline for future research in the sea target detection from GNSS-R observables comes from the exploitation of DDM deconvolution methods in order to take advantage from the application of target detector to deconvolved GNSS-R data, e.g., the bistatic scattering coefficient. False alarms caused by noise or fast moving targets, such as airplanes, can be reduced by multiple-image approaches, for example, by further processing target maps related to consecutively acquired DDMs. Target location ambiguity is a key issue for the geolocation of the detected target, and further investigation is needed in order to achieve an unambiguous sea target detection in a geographical coordinate system. The target ambiguity problem is expected to benefit from this multilook approach as well by analyzing the two candidate target tracks in a geographic coordinate system. The future spaceborne GNSS-R missions, such as the current NASA CYGNSS mission, are expected to bring new data for additional performance assessment of the proposed algorithm and an evaluation of its competitiveness in the field of sea target detection from remote sensing imagery.

\section{ACKNOWLEDGMENT}

The authors would like to thank SSTL and the Measurement of Earth Reflected Radio-navigation Signals By Satellite (MeRRByS) project for the U.K. TDS-1 data provided to conduct this research at no cost.

\section{REFERENCES}

[1] S. Brusch, S. Lehner, T. Fritz, M. Soccorsi, A. Soloviev, and B. van Schie, "Ship surveillance with terra SAR-X," IEEE Trans. Geosci. Remote Sens., vol. 49, no. 3, pp. 1092-1103, Mar. 2011.

[2] A. Gambardella, F. Nunziata, and M. Migliaccio, "A physical fullresolution SAR ship detection filter," IEEE Geosci. Remote Sens. Lett., vol. 5 , no. 4, pp. 760-763, Oct. 2008.

[3] D. Robinson, K. Dewey, and R. Heim, "Global snow cover monitoring: An update," Bull. Amer. Meteorol. Soc., vol. 74, no. 9, pp. 1689-1696, 1993.

[4] "U.S. National Ice Center," Jan. 16, 2017. [Online]. Available: http://www.natice.noaa.gov/

[5] P. Iervolino, R. Guida, and P. Whittaker, "A model for the backscattering from a canonical ship in SAR imagery," IEEE J. Sel. Topics Appl. Earth Observ. Remote Sens., vol. 9, no. 3, pp. 1163-1175, Mar. 2016.

[6] U. Nations, "Review of maritime transport-2014," in Proc. United Nations Conf. Trade Develop., 2014.

[7] P. Whittaker, M. Cohen, D. Hall, and L. Gomes, "An affordable small satellite SAR mission," in Proc. 8th IAA Symp. Small Satellite Earth Observ., 2011, pp. 1-20.

[8] S. Ramongassie et al., "Radar and AIS sensors constellation for global maritime surveillance," in Proc. IEEE Int. Geosci. Remote Sens. Symp., 2010, pp. 3793-3796.
[9] G. Yang, B. Li, S. Ji, F. Gao, and Q. Xu, "Ship detection from optical satellite images based on sea surface analysis," IEEE Geosci. Remote Sens. Lett., vol. 11, no. 3, pp. 641-645, Mar. 2014.

[10] C. Zhu, H. Zhou, R. Wang, and J. Guo, "A novel hierarchical method of ship detection from spaceborne optical image based on shape and texture features," IEEE Trans. Geosci. Remote Sens., vol. 48, no. 9, pp. 3446-3456, Sep. 2010.

[11] D. J. Crisp, "The state-of-the-art in ship detection in synthetic aperture radar imagery," Intell., Surveillance and Reconnaissance Div., Inf. Sci. Lab., Def., Sci. Technol. Org., Edinburgh, SA, Australia, Res. Rep. DSTORR-0272, May 2004.

[12] P. Lombardo and M. Sciotti, "Segmentation-based technique for ship detection in SAR images," IEE Proc.-Radar, Sonar Navigat., vol. 148, no. 3, pp. 147-159, Jun. 2001.

[13] "Observing Systems Capability Analysis and Review Tool website, Sea-ice cover requirements," Jan. 16, 2017. [Online]. Available: https://www.wmo-sat.info/oscar/variables/view/135

[14] "COSMO-SkyMed Mission - COSMO-SkyMed System Description \& User Guide," Italian Space Agency (ASI). Jan. 16, 2017. [Online]. Available: http://www.e-geos.it/products/pdf/csk-user_guide.pdf

[15] C. Hall and R. R. Cordey, "Multistatic scatterometry," in Proc. Int. Geosci. Remote Sens. Symp., 1988, pp. 561-562.

[16] V. Zavorotny and A. Voronovich, "Scattering of GPS signals from the ocean with wind remote sensing applications," IEEE Trans. Geosci. Remote Sens., vol. 38, no. 2, pp. 951-964, Mar. 2000.

[17] M. P. Clarizia and C. S. Ruf, "Wind speed retrieval algorithm for the cyclone global navigation satellite system (CYGNSS) mission," IEEE Trans. Geosci. Remote Sens., vol. 54, no. 8, pp. 4419-4432, Aug. 2016.

[18] M. P. Clarizia, C. S. Ruf, P. Jales, and C. Gommenginger, "Spaceborne GNSS-R minimum variance wind speed estimator," IEEE Trans. Geosci. Remote Sens., vol. 52, no. 11, pp. 6829-6843, Nov. 2014.

[19] N. Rodriguez-Alvarez, D. M. Akos, V. U. Zavorotny, J. A. Smith, A. Camps, and C. W. Fairall, "Airborne GNSS-R wind retrievals using delay-Doppler maps," IEEE Trans. Geosci. Remote Sens., vol. 51, no. 1 , pp. 626-641, Jan. 2013.

[20] E. Valencia et al., "Ocean surface's scattering coefficient retrieval by delay-Doppler map inversion," IEEE Geosci. Remote Sens. Lett., vol. 8, no. 4, pp. 750-754, Jul. 2011.

[21] D. Schiavulli, F. Nunziata, G. Pugliano, and M. Migliaccio, "Reconstruction of the normalized radar cross section field from GNSS-R delayDoppler map," IEEE J. Sel. Topics Appl. Earth Observ. Remote Sens. vol. 7, no. 5, pp. 1573-1583, May 2014.

[22] M. P. Clarizia, C. Ruf, P. Cipollini, and C. Zuffada, "First spaceborne observation of sea surface height using GPS-Reflectometry," Geophys. Res. Lett., vol. 43, no. 2, pp. 767-774, Jan. 2016.

[23] E. Valencia, A. Camps, H. Park, and N. Rodriguez-Alvarez, X. BoschLluis, and I. Ramos-Perez, "Oil slicks detection using GNSS-R," in Proc. IEEE Int. Geosci. Remote Sens. Symp., Vancouver, BC, Canada, Jul. 2011, pp. 4383-4386.

[24] C. Li, W. Huang, and S. Gleason, "Dual antenna space-based GNSS-R ocean surface mapping: Oil slick and tropical cyclone sensing," IEEE J. Sel. Topics Appl. Earth Observ. Remote Sens., vol. 8, no. 1, pp. 425-435, Jan. 2015.

[25] Q. Yan and W. Huang, "GNSS-R delay-Doppler map simulation based on the 2004 Sumatra-Andaman tsunami event," J. Sens., vol. 2016, 2016, Art. no. 2750862.

[26] Q. Yan and W. Huang, "Tsunami detection and parameter estimation from GNSS-R delay-Doppler map," IEEE J. Sel. Topics Appl. Earth Observ. Remote Sens., vol. 9, no. 10, pp. 4650-4659, Oct. 2016.

[27] H. Carreno-Luengo et al., "3Cat-2-An experimental nanosatellite for GNSS-R earth observation: Mission concept and analysis," IEEE J. Sel. Topics Appl. Earth Observ. Remote Sens., vol. 9, no. 10, pp. 4540-4551, Oct. 2016.

[28] S. Gleason, "Space reflected GNSS signals detected off an ice sheet in an Alaskan sound and from a diverse land surface in the plains of north America," in Proc. GNSS-R Workshop, Surrey, U.K., Jun. 2005.

[29] A. Alonso Arroyo, V. U. Zavorotny, and A. Camps, "Sea ice detection using GNSS-R data from UK TDS-1," in Proc. IEEE Int. Geosci. Remote Sens. Symp., 2016, pp. 2001-2004.

[30] M. Wiehl, B. Legrésy, and R. Dietrich, "Potential of reflected GNSS signals for ice sheet remote sensing," Prog. Electromagn. Res., vol. 40, pp. 177-205, 2003.

[31] G. Carrie, T. Deloues, J. Mametsa, and S. Angelliaume, "Ship detection based on GNSS reflected signals: An experimental plan," in Proc.: Space Reflecto, Calais, France, 2011. 
[32] M. P. Clarizia, P. Braca, C. S. Ruf, and P. Willett, "Target detection using GPS signals of opportunity," in Proc. 18th Int. Conf. Inf. Fusion, Washington, DC, USA, 2015, pp. 1429-1436.

[33] W. Ji, C. Xiu, W. Li, and L. Wang, "Ocean surface target detection and positioning using the spaceborne GNSS-R delay-Doppler maps," in Proc. IEEE Int. Geosci. Remote Sens. Symp., Quebec City, QC, Canada, 2014, pp. 3806-3809.

[34] Y. Lu, D. Yang, W. Li, J. Ding, and Z. Li, "Study on the new methods of ship object detection based on GNSS reflection," Marine Geodesy, vol. 32, no. 1, pp. 22-30, 2013.

[35] C. Kabakchiev, I. Garvanov, V. Behar, and H. Rohling, "The experimental study of possibility for radar target detection in FSR using L1-based noncooperative transmitter," in Proc. 14th Int. Radar Symp., Jun. 19-21, 2013, vol. 2, pp. 625-630.

[36] Univ. Michigan, "CYGNSS," Sep. 16, 2016. [Online]. Available: http://clasp-research.engin.umich.edu/missions/cygnss/science.php.

[37] R. Olive, A. Amezaga, H. Carreno-Luengo, H. Park, and A. Camps, "Implementation of a GNSS-R payload based on software-defined radio for the 3CAT-2 mission," IEEE J. Sel. Topics Appl. Earth Observ. Remote Sens. vol. 9 , no. 10 , pp. 4824-4833, Oct. 2016.

[38] NOAA, "Shoreline database," Sep. 16, 2016. [Online]. Available: https://www.ngdc.noaa.gov/mgg/shorelines/

[39] M. Tello, C. Lopez-Martinez, and J. J. Mallorqui, "A novel algorithm for ship detection in SAR imagery based on the wavelet transform," IEEE. Geosci. Remote Sens. Lett., vol. 2, no. 2, pp. 201-205, Apr. 2005.

[40] A. Marino, M. Sugimoto, K. Ouchi, and I. Hajnsek, "Validating a notch filter for detection of targets at sea with ALOS-PALSAR data: Tokyo bay," IEEE J. Sel. Topics Appl. Earth Observ. Remote Sens., vol. 7, no. 12, pp. 4907-4918, Dec. 2014.

[41] M. A. Richards, J. A. Scheer, and W. A. Holm, Principles of Modern Radar: Basic Principles. Edison, NJ, USA: SciTech, 2010.

[42] H. Park, A. Camps, D. Pascual, R. Onrubia, A. Alonso-Arroyo, and F. Martin, "Evolution of PAU/PARIS end-to-end performance simulator (P2EPS) towards GNSS reflectometry, radio occulation and scatterometry simulator (GEROS-SIM)," in Proc. IEEE Geosci. Remote Sens. Symp., Milan, Italy, 2015, pp. 4757-4760.

[43] H. Park et al., "Retracking considerations in spaceborne GNSS-R altimetry," GPS Solution, vol. 16, no. 4, pp. 507-518, Jan. 2012.

[44] J. F. Marchan-Hernandez, A. Camps, N. Rodriguez-Alvarez, E. Valencia, X. Bosch-Lluis, and I. Ramos-Perez, "An efficient algorithm to the simulation of delay-Doppler maps of reflected global navigation satellite system signals," IEEE Trans. Geosci. Remote Sens., vol. 47, no. 8, pp. 2733-2740, Aug. 2009.

[45] H. Park, E. Valencia, N. Rodriguez-Alvarez, X. Bosch-Lluis, I. Ramos-Perez, and A. Camps, "New approach to sea surface wind retrieval from GNSS-R measurements," in Proc. IEEE Int. Geosci. Remote Sens. Symp., 2011, pp. 1469-1472.

[46] M. Clarizia et al., "Analysis of GNSS-R delay-Doppler maps from the UK-DMC satellite over the ocean," Geophys. Res. Lett., vol. 36, no. 2, Jan. 2009, Art. no. L02608.

[47] S. Gleason et al., "Detection and processing of bistatically reflected GPS signals from low-earth orbit, for the purpose of ocean remote sensing," IEEE Trans. Geosci. Remote Sens., vol. 43, no. 6, pp. 1229-1241, Jun. 2005.

[48] N. Rodriguez-Alvarez and J. Garrison, "Recent advances in retrieval of ocean surface wind fields from GNSS-R delay-Doppler maps," in Proc. ION 2015 Pacific PNT Meet., Honolulu, HI, USA, 2015, pp. 518-521.

[49] W. T. Labs, "GeoHack-Hibernia gravity base structure," Sep. 20, 2016. [Online]. Available: https://tools.wmflabs.org/geohack/ geohack.php?pagename $=$ Hibernia_Gravity_Base_Structure\&params = 46_45_1.57_N_48_46_58.54_W

[50] J. Maslanik and J. Stroeve, "Near-real-time DMSP SSMIS daily polar gridded sea ice concentrations, version 1," NASA National Snow and Ice Data Center Distributed Active Archive Center, Boulder, CO, USA, 1999. [Online]. Available: http://nsidc.org/data/nsidc-0081\#

[51] L. Guoyo, World Atlas of Oil and Gas Basins. Hoboken, NJ, USA: Wiley-Blackwell, 2011.

[52] T. S. Portal, "Number of offshore rigs worldwide as of 2015, by region," Sep. 21, 2016. [Online]. Available: https://www.statista.com/ statistics/279100/number-of-offshore-rigs-worldwide-by-region/

[53] K. Eldhuset, "An automatic ship and ship wake detection system for spaceborne SAR images in coastal regions," IEEE Trans. Geosci. Remote Sens., vol. 34, no. 4, pp. 1010-1019, Jul. 1996.
[54] "TerraSAR-X Ground Segment Basic Product Specification Document," Feb. 2008. [Online]. Available: http://www.intelligenceairbusds.com/files/pmedia/public/r466_9_tx-gs-dd-3302_basic-productspecification-document_1_9.pdf

[55] "Sentinel-1 User Handbook," Sep. 2013. [Online]. Available: https:// sentinel.esa.int/documents/247904/685163/Sentinel-1_User_Handbook

[56] “SPOT 6 \& SPOT 7 Imagery User Guide," July 2013. [Online]. Available: https://www.spaceoffice.nl/blobs/Dataportaal/User_Guide_SPOT6_V1.0. pdf

[57] "Landsat 7 User Guide," 1998. [Online]. Available: http:// landsathandbook.gsfc.nasa.gov/pdfs/Landsat7_Handbook.pdf

[58] "Sentinel-2 User Handbook," Jul. 2015. [Online]. Available: https:// sentinel.esa.int/documents/247904/685211/Sentinel-2_User_Handbook

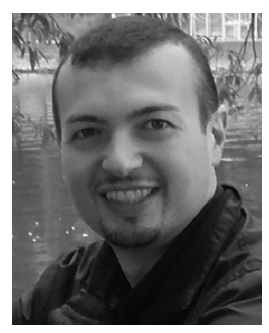

Alessio Di Simone was born in Torre del Greco, Naples, Italy, on September 11, 1989. He received the B.Sc. and M.Sc. Laurea degrees (cum laude) in telecommunication engineering and the Ph.D. degree in information technology and electrical engineering from the University of Naples "Federico II," Naples, in 2011, 2013, and 2017, respectively.

In May 2017, he joined the NATO Science and Technology Organization Centre for Maritime Research and Experimentation, La Spezia, Italy, as a Research Fellow in the framework of the "Ship Detection/Tracking using Multistatic GPS Signals" project funded by the U.S. Office of Naval Research. His main research interests are in the field of microwave remote sensing and electromagnetics: modeling of the electromagnetic scattering from natural surfaces, synthetic aperture radar (SAR) and global navigation satellite systems-reflectometry (GNSS-R) data simulation, information retrieval, SAR despeckling, maritime surveillance and target detection/tracking using GNSS signals of opportunity.

Dr. Di Simone was awarded a prize for the Best Master Thesis in Remote Sensing by the IEEE South Italy Geoscience \& Remote Sensing Chapter in 2015 .

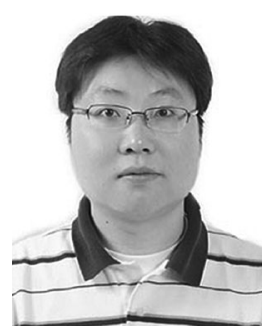

Hyuk Park (S'05-AM'09-M'12-SM'15) was born in South Korea. He received the B.S. degree in mechanical engineering from the Korea Advanced Institute of Science and Technology, Daejeon, South Korea, in 2001, and the M.S. and Ph.D. degrees in information and mechatronics from Gwangju Institute of Science and Technology, Gwangju, South Korea, in 2003 and 2009, respectively.

In 2009, he joined the Remote Sensing Group, Polytechnic University of Catalonia (UPC), Barcelona, Spain, as a Postdoctoral Researcher. In 2011, he was a grant holder of National Research Foundation funded by the Korean Government. Since 2012, he has been a Research Associate with a Juan de la Cierva grant funded by the Spanish Ministry of Economy and Competitiveness. He is currently with the Passive Remote Sensing Group, UPC, for satellite remote sensing for microwave radiometry and global navigation satellite systems-reflectometry. His main research interests are in the area of remote sensing, particularly passive microwave remote sensing, including system design, modeling and simulation, and image processing. 


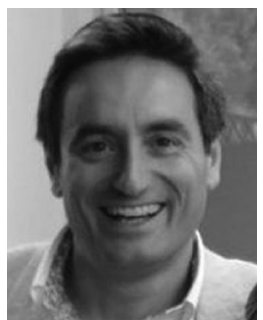

Daniele Riccio (M'91-SM'99-F'14) was born in Naples, Italy. He received the Laurea degree (cum laude) in electronic engineering from the University of Naples Federico II, Naples, Italy, in 1989.

His career has been developed at the University of Naples Federico II, where he is currently a Full Professor of electromagnetic theory and remote sensing with the Department of Electrical Engineering and Information Technology. Additionally, he was a Research Scientist with the Italian National Research Council, Institute for Research on Electromagnetics and Electronic Components (1989-1994), a Guest Scientist with the German Aerospace Centre (DLR), Munich, Germany (1994 and 1995), and a Lecturer to the Ph.D. program with the Universitat Politecnica de Catalunya, Barcelona, Spain (2006) and with the Czech Technical University, Prague, Czech Republic (2012). He is also a member of the Cassini Radar Science Team, the Principal Investigator for international research projects on exploitation of remote sensing data and design of synthetic aperture radars, and participates to technical committees of international symposia on electromagnetics and remote sensing. $\mathrm{He}$ has authored three books, including Scattering, Natural Surfaces and Fractals (New York, NY, USA: Academic, 2007), and more than 400 scientific papers. Heis the Coordinator of the Ph.D. School in Information Technology and Electrical Engineering at the University of Napoli Federico II. His research interests include microwave remote sensing, electromagnetic scattering, synthetic aperture radar with emphasis on sensor design, data simulation and information retrieval, as well as application of fractal geometry to remote sensing.

Prof. Riccio serves as an Associate Editor for some journals on Remote Sensing. He received the 2009 Sergei A. Schelkunoff Transactions Prize Paper Award for the best paper published in the IEEE TRANSACTIONS ON ANTENNAS AND PROPAGATION in 2008.

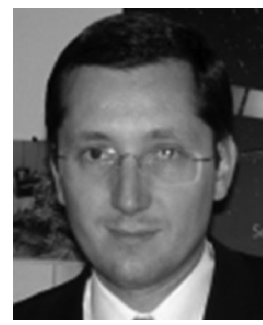

Adriano Camps (S'91-A'97-M'00-SM'03-F'11) was born in Barcelona, Spain, in 1969. He received the Degree in telecommunications engineering and $\mathrm{Ph} . \mathrm{D}$. degree in telecommunications engineering from the Universitat Politècnica de Catalunya (UPC), Barcelona, Spain, in 1992 and 1996, respectively.

In 1991 to 1992, he was at the ENS des Télécommunications de Bretagne, France, with an Erasmus Fellowship. Since 1993, he has been with the Electromagnetics and Photonics Engineering Group, Department of Signal Theory and Communications, UPC, where he was first Assistant Professor, became an Associate Professor in 1997, and has been a Full Professor since 2007. He has authored or coauthored more than 160 journal papers in peer-reviewed journals and more than 350 international conference presentations. He holds ten patents. His research interests are focused on: 1) microwave remote sensing, with special emphasis in microwave interferometric radiometry by aperture synthesis (ESA's SMOS mission); 2) passive microwave remote sensing using signals of opportunity (GNSS-Reflectometry); and, more recently, 3) the use of nanosatellites as costeffective platforms to test innovative concepts for Earth Observation, such as the 3Cat-2, a 6U-class cubesat mission launched in August 2016 carrying on-board an innovative GNSS-R payload. 